\title{
A STUDY OF GRAVITATIONAL EFFECTS ON SINGLE ELONGATED VAPOR BUBBLES
}

\author{
Alex Scammell \\ Department of Mechanical Engineering \\ University of Maryland, College Park, 20740, USA \\ Email: ascammel@umd.edu \\ Jungho Kim* \\ Department of Mechanical Engineering
}

3137 Glenn L. Martin Hall, Building 088, University of Maryland, College Park, 20740, USA

Email:kimjh@umd.edu; Telephone: 301-405-5437

\section{Mirco Magnini}

Heat and Mass Transfer Laboratory (LTCM)

École Polytechnique Fédérale de Lausanne (EPFL), EPFL-STI-IGM-LTCM, Station 9, Lausanne

1015, Switzerland

Email:mirco.magnini@epfl.ch

\author{
John Thome \\ Heat and Mass Transfer Laboratory (LTCM) \\ École Polytechnique Fédérale de Lausanne (EPFL), EPFL-STI-IGM-LTCM, Station 9, Lausanne \\ 1015, Switzerland \\ Email: john.thome@epfl.ch \\ *corresponding author
}

\section{ABSTRACT}

An experimental study was completed on the heat transfer induced by a single elongated vapor bubble rising in vertical, upward, co-current flow at various accelerations. The bubble shape and behavior were also studied under those conditions. Tests were conducted using a flow boiling rig which coupled flow visualization with measurements of the heat transfer and liquid film thickness. The bubble drift velocity was shown to dictate the bubble shape as well as the relative influences of thin film evaporation and turbulent wake heat transfer. When present, vortices shed behind the bubble tail created peaks in the heat transfer coefficient whose magnitude decreased with increasing liquid Reynolds number and acceleration. The vortex frequency was found to be linearly dependent on plunging velocity. A criteria for vortex incipience was subsequently proposed. 


\section{INTRODUCTION}

Slug flow, characterized by elongated bubbles separated by liquid slugs, is seen in many groundand space-based applications such as nuclear reactors, cryogenic transfer lines, and compact heat exchangers. These systems require successful prediction of the regime's heat removal capability to operate in a safe and efficient manner. Mechanistic modeling provides a cost-effective means to estimate slug flow heat transfer under various design conditions, but experiments are ultimately needed for model guidance and validation. Specifically, local measurements of the flow field and heat transfer in the presence of a single elongated bubble can be viewed as a "unit cell" for the broader regime of slug flow and incorporated into model development.

\subsection{Taylor bubble hydrodynamics}

For the vertical, upward co-current flow configuration, the bubble shape is dictated by the ratio

of buoyancy to surface tension forces as described by the Bond number, $B o=\frac{\left(\rho_{l}-\rho_{v}\right) g D^{2}}{\sigma}$. Slug flows with $B o>40$ are considered macro-scale [1] and exhibit bullet-shaped bubbles, also known as Taylor bubbles. This regime has been widely studied; a review of the literature prior to 1992 is given in Fabre and Line [2] which summarizes fundamental aspects of the bubble hydrodynamics. Nicklin et al. [3] proposed a correlation for bubble velocity, $U_{b}$, of the form,

$$
U_{b}=C U_{l}+U_{b, 0}
$$

where $C$ is a constant that defines the bubble rise velocity depending on the fluid velocity profile, $U_{l}$ is the mean background liquid velocity, and $U_{b, 0}$ is the bubble rise velocity in a stagnant fluid column. Values of $C$ were theoretically and experimentally determined to be 2 and 1.2 for a Taylor bubble flowing within fully developed laminar and turbulent flows, respectively $[3,4]$. White and Beardmore [5] proposed a graphical relation for $U_{b, 0}$ based on experimental measurements of air bubbles in a variety of stagnant liquids. Results were broken into several regimes depending on the relative influence of inertial, surface tension, and viscous forces acting on the bubble. Brown [6] modified the potential flow solution for the flow field around rising Taylor bubbles to account for liquid viscosity and developed a correlation that agreed well with experimental results when viscous forces could be ignored at the bubble nose. Viana et al. [1] combined bubble velocity data from the literature with their own data to create a universal 
correlation for $U_{b, 0}$ based on $B o$ and a buoyancy Reynolds number similar to the Grashof number. Rattner and Garimella [7] recently completed an experimental study on intermediate scale slug flows and proposed an extension of Bendiksen's [8] prediction for $U_{b, 0}$.

As the bubble rises, a liquid film is formed between the liquid/vapor interface and the tube wall that thins as it moves towards the tail, eventually reaching a constant thickness $(\delta)$ where the liquid velocity profile within the film has become fully developed. Campos and Carvalho [9] proposed an equation to calculate the distance from the nose, $L_{f}$, at which the film stabilizes. Nogueira et al. [10] compared experimentally measured values of $L_{f}$ to predictions by Campos and Carvalho and found that the agreement was heavily dependent on the measured film Reynolds number, $R e_{U_{\delta}}=\frac{U_{\delta} \delta}{v}$, where $U_{\delta}$ is the mean velocity in the film. $L_{f}$ was underpredicted at film Reynolds numbers below 40, but overpredicted above $R e_{U_{\delta}}=80$.

Prediction of the fully developed film thickness has been the subject of both theoretical and experimental work. Goldsmith and Mason [11] developed a relation for $\delta$ by solving the NavierStokes equations for a laminar thin film around a Taylor bubble. The thin film assumption was later relaxed by Brown [6] to obtain similar results. Llewellin et al. [12] approximated $\delta$ for air bubbles rising in a variety of Newtonian fluids by correlating the bubble volume to its length. It was found that the relative film thickness $(\delta / R)$ was solely a function of the non-dimensional parameter $N_{f}=\frac{\sqrt{g D^{3}}}{v}$ (this is also equal to the square root of the Galileo number, $G a$ ). An empirical correlation was proposed based on the collected data as well as that of Nogueira et al. [10].

At the tail, the liquid film plunges into the liquid slug trailing the bubble creating vortices in the wake whose structures vary in complexity from organized toroids to chaotic shedding. Characterization of the flow field in this region has seen significant progress in recent years due in large part to the advancement of experimental techniques. Campos and Carvalho [9] performed a photographic study of rising air bubbles in stagnant water/glycerol mixtures seeded with dye to determine the structure of the bubble wake. Three wake patterns were identified. For $N_{f}<500$ the wake exhibited a laminar, closed, axisymmetric, toroidal vortex with internal recirculatory flow. A transition region occurred for $500<N_{f}<1500$ where the toroidal vortex remained intact, but became non-axisymmetric due to oscillations in the bubble tail. For $N_{f}>1500$, the vortices in the wake were shed randomly, exhibiting turbulent characteristics 
that decayed as they moved downstream. Liu et al. [13] compared these boundaries to wake velocity profiles for vapor bubbles rising in stagnant liquid nitrogen. Results from their particle image velocimetry (PIV) technique revealed that due the significant property differences between water (used by Campos and Carvalho) and liquid nitrogen, the prediction of wake regimes was not applicable to cryogenics as transitional and laminar wake patterns were observed for $N_{f} \gg 1500$. Pinto et al. [14] redefined Campos and Carvalho's criteria in terms of a Reynolds number based on the relative velocity between the bubble and liquid, $R e_{U_{d}}=\frac{\rho_{l} U_{d} D}{v}$ where $U_{d}=U_{b}-U_{l}$. They proposed that the wake would be fully turbulent for $R e_{U_{d}}>525$ in the case of liquid flowing co-currently with the bubble, but noted that the limit had not been experimentally confirmed. Kawaji et al. [15] observed turbulent bubble wake characteristics and suggested the vortices were due to a Helmholtz-type instability resulting from the relative velocity difference between the plunging film and the trailing slug. They found that the distance behind the bubble at which the film became unstable (the "penetration length") remained relatively constant with respect to bubble length once the flow within the film became fully developed. Shemer et al. [16] used PIV to study the velocity profiles in the wake of single bubbles rising in both laminar and turbulent background flows. They found the wake flow field could be effectively turbulent even when the base flow was laminar. Turbulence quantities calculated from the data showed that the initial mixing process occurred in the near wake region and persisted a few diameters downstream of the bubble tail.

The flow field around a Taylor bubble can be viewed in two reference frames, a laboratory reference frame where the bubble rises in a stationary tube and a bubble reference frame which moves with the bubble as shown in Figure 1. This assumes the bubble does not grow as it rises in the tube. In this paper, unprimed and primed quantities refer to area average velocities in the laboratory and bubble reference frames, respectively. It should be noted that the drift velocity, $U_{d}=U_{b}-U_{l}=U_{b}^{\prime}-U_{l}^{\prime}$, is independent of the reference frame as is the plunging velocity, $U_{p}=U_{f}+U_{l}=U_{f}^{\prime}-U_{l}^{\prime}$. The average film velocity, $U_{f}$, can be calculated from a mass balance and the film thickness:

$$
U_{f}=\frac{\left(U_{l}-U_{b}\right) R^{2}}{\left(2 R \delta-\delta^{2}\right)}-U_{b}
$$




\section{Laboratory Reference Frame}

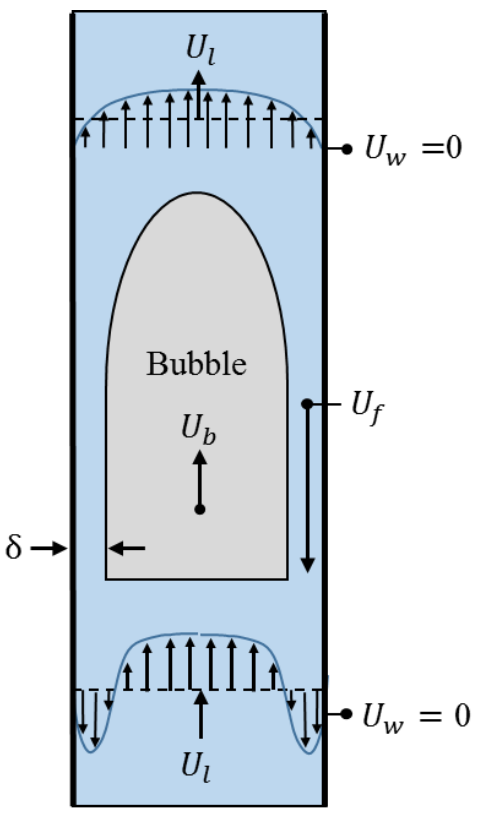

Bubble Reference Frame

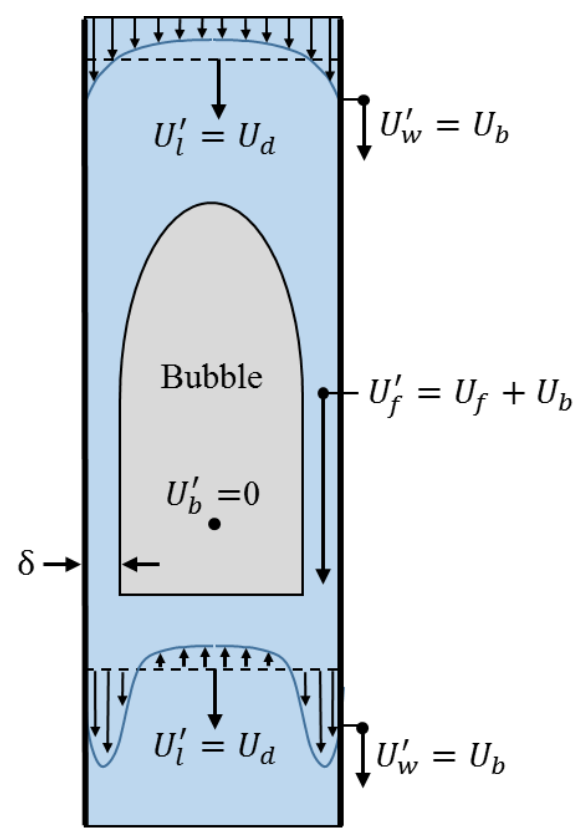

Figure 1: Schematic of velocity profiles ahead of the bubble and in the near wake region in the laboratory (left) and bubble (right) reference frames. Note that the velocity vector lengths are not to scale.

\subsection{Taylor bubble heat transfer}

The bubble shape and surrounding liquid hydrodynamics have a significant impact on the wall heat transfer. The experimental work to date has largely focused on measuring the overall heat transfer enhancement due to Taylor bubble trains with respect to single-phase flow rather than on the local mechanisms of heat transfer around each bubble. Hetsroni and Rozenblit [17] briefly explored the mechanistic approach using a thin, black heated film installed within a tube to make temperature measurements using an infrared (IR) camera. The wall temperature during the passage of a Taylor bubble was observed to be higher compared to the liquid slug behind the bubble. This suggests a higher heat transfer coefficient in the wake than in the liquid film, but no quantitative data was presented. Babin et al. [18-20] investigated the wake heat transfer enhancement for single air bubbles rising in stagnant and vertically flowing water using a similar IR technique. They observed that the heat transfer coefficient rose rapidly just behind the bubble tail before decaying to the original single-phase value far downstream. Bubbles moving cocurrently within turbulent flow created smaller heat transfer enhancement than those moving 
within laminar flow. Heat transfer measurements were compared to PIV measurements obtained by Shemer et al. [16] to show that the growth and decay of velocity fluctuations in the wake may correspond to the heat transfer coefficient profile observed for turbulent background flows. Scammell and Kim [21] observed the heat transfer profile around single rising vapor Taylor bubbles in a silicon tube using IR thermography. Heat transfer enhancement was found to occur in the bubble wake where turbulent vortices caused mixing in the trailing liquid. The wake heat transfer profile peaked at approximately one diameter behind the bubble tail then decreased towards the original single-phase value as the bubble passed. A decrease in the peak wake heat transfer coefficient with increasing liquid velocity was attributed to reduced residence time of the bubble over a particular tube location. The velocity and frequency of wake vortex shedding were also analyzed.

\subsection{Capillary bubble heat transfer}

The advent of micro-scale (defined as $B o<0.9-19.7$ by [5,8,22-24]) heat exchangers has spurred work into the behavior of micro-channel slug flow. Slug flow under these conditions is characterized by elongated bubbles, also known as capillary bubbles, with near-spherical caps at the nose and the tail. Several modeling efforts and numerical studies of local heat transfer around capillary bubbles have been completed. A model by Jacobi and Thome [25] featured a two-zone representation of evaporation for elongated bubble flows where the regime was divided into a liquid slug region and a thin film region. It was suggested that the main mechanism of heat transfer was evaporation of the thin film trapped between the bubble and the heated wall. This model was modified by Thome et al. [26] to include three zones: a liquid slug, an evaporating elongated bubble, and a vapor slug. As with the two-zone model, evaporation in the thin liquid film provided heat transfer several orders of magnitude higher than the single-phase heat transfer due to the liquid slug.

Magnini et al. [27] numerically computed the shape, length, and local heat transfer of elongated bubbles during boiling of several fluids in a microchannel. They found that as the bubble entered a heated channel containing a developing thermal boundary layer, evaporation of the liquid film removed heat from the fluid and caused the heat transfer to become larger than for single-phase flow. The heat transfer coefficient rose monotonically from the bubble nose towards

the tail with the highest values occurring in the bubble wake region. Based on these results, they 
modified the three-zone model by Thome et al. [26] to include unsteady conduction through the liquid, and obtained better agreement with the simulations.

\subsection{Objective}

The objective of the current work is to utilize the variable gravity environment aboard an aircraft flying parabolic trajectories to study the heat transfer due to a single bubble in a heated tube at various $B o$. The data can be used to identify the applicability of current slug flow heat transfer models for reduced gravity and near-critical fluid conditions.

\section{EXPERIMENTAL APPARATUS}

To characterize the heat transfer and dynamics of rising elongated bubbles, a flow boiling experiment was conducted in which measurements of the local wall heat transfer and high speed images were obtained as single bubbles of varying length rose in a vertical column containing upward flowing liquid. The experimental facility is summarized below and is described in detail by Scammell and Kim [21] and Kim et al. [28]. A study of single bubbles was chosen in lieu of bubble trains so flow conditions upstream and downstream of the bubbles could be measured, reducing the complexity in approximating the flow patterns and understanding the heat transfer profile around each bubble.

\subsection{Flow loop}

A schematic of the experiment flow loop is shown in Figure 2. The working fluid was 3M Novec HFE $7100\left(\mathrm{C}_{4} \mathrm{~F}_{9} \mathrm{OCH}_{3}\right)$, a non-toxic, dielectric fluid with a normal boiling temperature of $60^{\circ} \mathrm{C}$. HFE 7100 was pumped in a subcooled liquid state using a gear pump (Micropump L21755) and the flow rate was measured by a turbine flowmeter (Omega FLR1009). The liquid was heated to near saturation at the test section inlet using a stainless steel preheater powered by a modified computer power unit (Silverstone SST-ST1000-P) and controlled using pulse width modulation via a LabVIEW ${ }^{\mathrm{TM}}$ interface.

The fluid then entered a section of the flow loop designed to create and release single Taylor bubbles. This section consisted of a bubble generation segment and a bypass segment connected at the downstream end by a three-way valve. To generate a bubble, the valve was set to divert the liquid flow through the bypass segment, while a wire heater evaporated liquid in the bubble 
generation segment. The bubble volume was varied by adjusting the power to the wire and the heating time. Once the desired bubble volume was generated, it was released by rotating the valve such that liquid was redirected through the bubble generation segment, pushing the bubble into the test section.

The bubble rose vertically into a $6 \mathrm{~mm}$ ID silicon test section positioned $170 \mathrm{~mm}$ downstream of the three-way valve where heat transfer measurements and flow visualization were made. Pressure taps were located at the inlet and outlet of silicon tube so differential and absolute pressures could be measured. The absolute pressure transducer (Omega PX209-030A5V) was used to determine the saturation temperature of the fluid entering the test section. Immediately after leaving the heated silicon tube, the rising bubble passed through a glass adiabatic section $380 \mathrm{~mm}$ downstream of the three-way valve where video was obtained using a CMOS video camera (Sentech STC-MBCM200U3V) at 505 frames per second. The high-speed visualization was used to determine the bubble length, study the dynamics of the tail, and analyze the bubble shape. The liquid film thickness was measured using two techniques: a laser displacement sensor (Keyence LK-G5000) and high-speed image analysis. The Keyence sensor was calibrated by measuring a known thicknesses of HFE 7100 within a maximum uncertainty of $17 \mu \mathrm{m}$. Due to movement of the sensor relative to the tube when the test apparatus was flown on the aircraft, data from the Keyence sensor could not be obtained. The film thickness under these conditions were therefore calculated from analysis of the high-speed video captured in the adiabatic section. Error in the measurement created by optical distortion was removed by a calibration procedure similar to Liu et al. [13] in which a grid of known spacing was placed in the test section tube. The apparent spacing was compared to the known spacing to create a calibration curve. The uncertainty associated with this technique was estimated to be $25 \mu \mathrm{m}$.

Bubbles were condensed and the liquid subcooled in a counterflow heat exchanger where the secondary fluid was cold water. A bellows-type accumulator was included after the condenser with the dry side open to the ambient to maintain the system pressure at nominally 1 bar, or the aircraft cabin pressure. Before re-entering the gear pump, the fluid was sent through a de-gassing membrane (Liqui-Cel SuperPhobic). A vacuum pump connected to the membrane for several hours prior to data being collected was used to degas the liquid.

All transducer data (pressure, temperature, flow, and test section input power) was collected using a 24-channel data acquisition system (Omega OMB-DAQ-3000) and recorded at a rate of 
$100 \mathrm{~Hz}$ through a LabVIEW ${ }^{\mathrm{TM}}$ interface. T-type thermocouples were installed at various locations in the flow loop for data analysis, calibration, and safety purposes. Uncertainty for the thermocouples was calculated to be $\pm 0.12^{\circ} \mathrm{C}$. Heat transfer measurements and flow visualization in the heated section were made using an IR camera (Electrophysics Silver 660M) at a frame rate of $246 \mathrm{~Hz}$. Typical uncertainties for the major instrumentation and some of the reduced parameters are summarized in Table 1.

Table 1: Typical uncertainties for important system parameters and measurements.

\begin{tabular}{lc} 
Parameter & Uncertainty \\
\hline$G\left[\mathrm{~kg} / \mathrm{m}^{2} \mathrm{~s}\right]$ & 5.2 \\
$P_{a b s}[\mathrm{millibar}]$ & 1.3 \\
$T_{I R}\left[{ }^{\circ} \mathrm{C}\right]$ & 0.14 \\
$k_{p}[\mathrm{~W} / \mathrm{m}-\mathrm{K}]$ & 0.01 \\
$\alpha_{S i}\left[\mathrm{~m}^{-1}\right]$ & 6.5 \\
$\alpha_{p}\left[\mathrm{~m}^{-1}\right]$ & 192 \\
$T_{s a t}\left[{ }^{\circ} \mathrm{C}\right]$ & 0.14 \\
$\delta_{K}[\mu \mathrm{m}]$ & 17 \\
$\delta_{V}[\mu \mathrm{m}]$ & 25
\end{tabular}




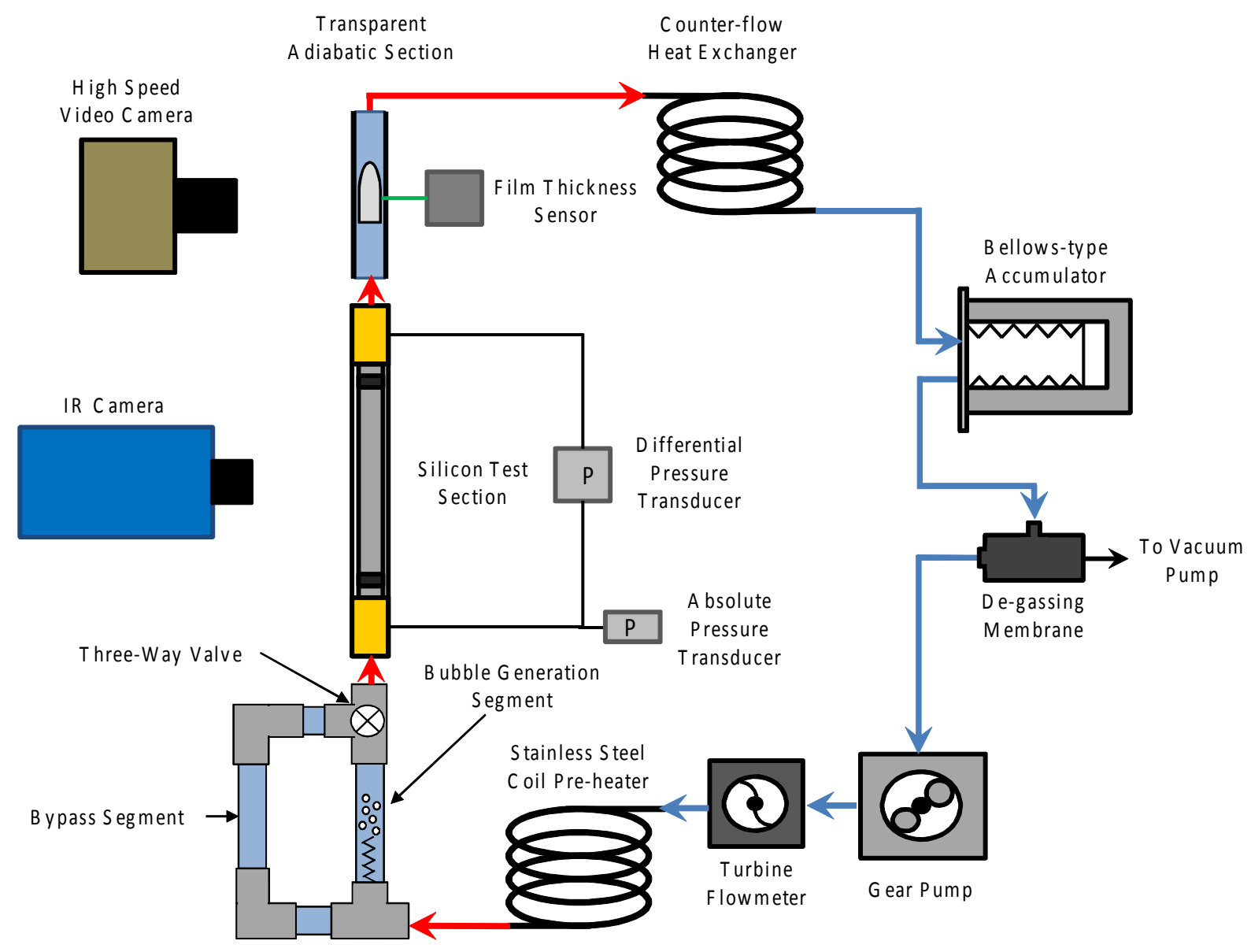

Figure 2: Functional Diagram for experimental test rig.

\subsection{IR technique}

Heat transfer measurements and flow visualization were obtained using an IR thermometry technique developed by Kim et al. [28] that exploits the transparency of silicon in the mid-IR range $(3-5 \mu \mathrm{m})$. HFE 7100 flowed through a $6 \mathrm{~mm}$ ID (8 mm OD) single crystal silicon tube which was coated on the inner wall with a $57 \mu \mathrm{m}$ layer of polyimide tape $(k=0.12 \mathrm{~W} / \mathrm{m}-\mathrm{K})$ as shown in Figure 3. One half of the inner circumference was covered with a $6 \mu \mathrm{m}$ thick layer of IR opaque paint containing carbon black (Nazdar GV111), which allowed the effective inner wall temperature to be measured through the silicon and polyimide layer. Two strips of the painted polyimide tape were also attached to the outer wall of the tube so the outer wall temperature could be measured. The silicon was doped to allow for resistive heating of the tube. The input power to the tube was varied by adjusting the voltage of a high-voltage power supply. 
A coupled conduction/radiation problem was solved which accounted for absorption, emission, and reflection of thermal energy from the various layers and the surroundings to determine the transient temperature profiles within the multilayer. The heat flux and heat transfer coefficient were then calculated for every camera pixel along the axial length of the tube. Details of the data reduction procedure can be obtained in Kim et al. [28]. To complement the heat transfer measurements, the flow was visualized using a set of six gold-plated mirrors (Figure 3) arranged such that flow visualization using IR and heat transfer measurements could be captured using a single camera.
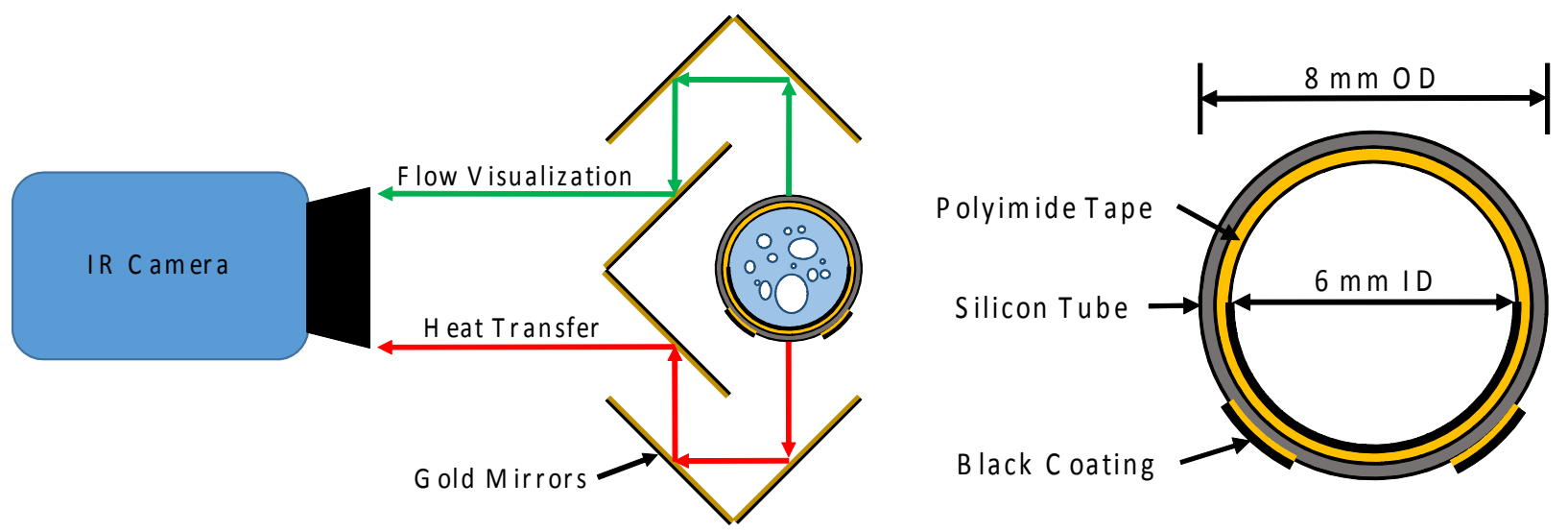

Figure 3: Mirrors to provide simultaneous heat transfer measurements and flow visualization (left image), and cross-sectional view of silicon tube with coated polyimide tape (right image).

The heat transfer measurements were validated using a series of $1 g$ single-phase and twophase experiments. Results presented in [21] showed good agreement between turbulent singlephase measurements and a correction factor proposed by Al-Arabi [29] for thermal entry length. Two-phase heat transfer coefficients agreed with data from a similar experiment and correlations over a wide range of vapor qualities [30]. Additional validation of the technique for the case of laminar single-phase flow was completed for this paper by measuring the temperature of the tube inner wall at $G=50 \mathrm{~kg} / \mathrm{m}^{2} \mathrm{~s}, R e_{l}=790, T_{i n}=45^{\circ} \mathrm{C}$, and applied heat fluxes of $2.0 \mathrm{~kW} / \mathrm{m}^{2}$ and 3.6 $\mathrm{kW} / \mathrm{m}^{2}$. Heat losses attributed to natural convection on the tube outer wall and axial conduction along the tube into the test section end caps were calculated to be $32 \pm 4 \%$ ( $23 \pm 3 \%$ to axial conduction, $9 \pm 1 \%$ to natural convection). Losses due to axial conduction were approximated using the temperature gradient at the downstream end of the tube, measured using the infrared 
technique. Natural convection losses were estimated using a correlation by Churchill and Chu [31] for free convection from a vertical wall, where the surface temperature was taken as the average outer wall temperature of the tube. An energy balance on the test section conducted by measuring the inlet and outlet liquid temperatures during heating found losses to be $35 \pm 12 \%$, supporting the calculations.

The ratio of Grashof number $\left(G r=\frac{g \beta\left(T_{w}-T_{m}\right) D^{3}}{v^{2}}\right)$ to the square of the liquid Reynolds number was of the range $0.68 \leq \frac{G r}{R e_{l}^{2}} \leq 1.17$, indicating that natural convection and forced convection may contribute to heat transfer from the tube wall, according to Incropera et al. [32]. In the absence of available correlations from the literature, Incropera et al. suggest that the mixed convection Nusselt number $\left(N u_{M}\right)$ be computed using the form $N u_{M}^{n}=N u_{F}^{n}+N u_{N}^{n}$, where $N u_{F}$ is the forced convection Nusselt number and $N u_{N}$ is the natural convection Nusselt number, both computed using existing correlations for the specific geometry. In this case, $N u_{F}$ was approximated from Shah and London [33] and $N u_{N}$ from Davis and Perona [34]. It was noted that the best correlation of data is often obtained for $n=3$, which was chosen for this case. Accounting for heat losses, the predicted and measured inner wall temperature $\left(T_{w}\right)$ offset by the tube inlet temperature $\left(T_{i n}\right)$ is plotted as a function of axial position in Figure 4 showing relatively good agreement within the uncertainty of the measurements and predictions. The decline in wall temperature at the tube exit is dominated by wall axial conduction losses.

Due to limitations in microgravity and hypergravity duration, extensive validation of the experiment was not able to be conducted under those conditions. However, energy balance tests, as described above, were repeated in the laboratory based on the conditions experienced on the aircraft. Notably, the effect of natural convection variation on the outer tube wall could not be studied, but two comments in this regard may be made. First, according to the correlation of Churchill and Chu [31], the average heat transfer coefficient for free convection over a vertical plate is related to gravity by the $1 / 4^{\text {th }}$ power. As a result, an increase in gravity level by a factor of 1.8 (hypergravity) yields an increase in the heat transfer coefficient by $16 \%$, yet only an overall increase in power lost by $1.4 \%(9 \% \times 1.16=10.4 \%)$. Second, natural convection loss for $a / g=0.01$ was assumed to be similar to $a / g=1$, given that the data collected under microgravity was taken $2-3$ seconds after the transition from $a / g=1.8$ to $a / g=0.01$ and 
currents associated with free convection may have still existed. All data was processed using the corresponding heat loss calculations for that gravity level.

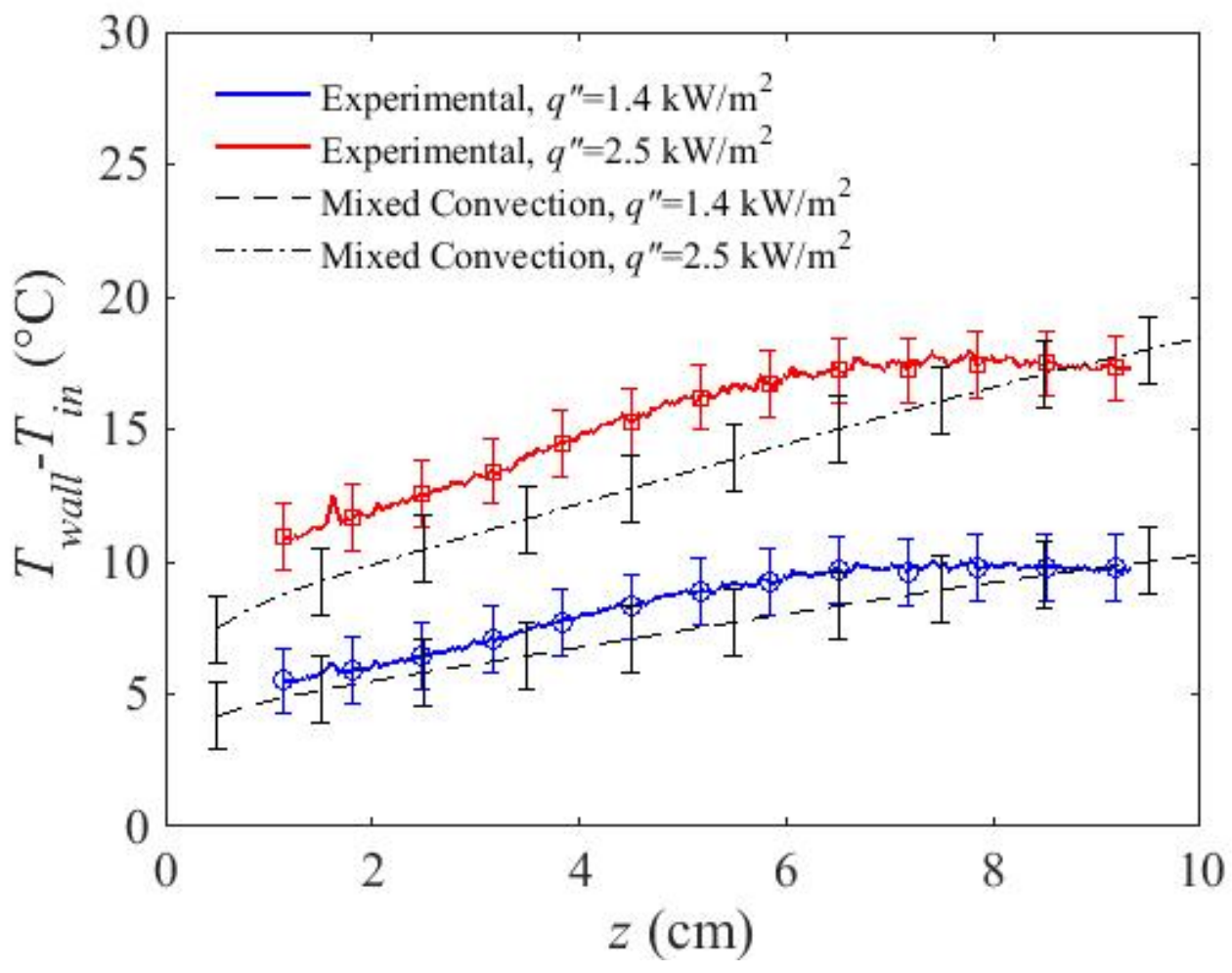

Figure 4: Experimental tube inner wall temperature compared to predictions for mixed convection for the parameters: $G=50 \mathrm{~kg} / \mathrm{m}^{2} \mathrm{~s}, R e=790, T_{i n}=45^{\circ} \mathrm{C}$, and heat flux of $1.4 \mathrm{~kW} / \mathrm{m}^{2}$ and $2.5 \mathrm{~kW} / \mathrm{m}^{2}$.

\subsection{Uncertainty analysis}

The uncertainty associated with heat transfer measurements can be divided into two parts: bias error and random error. Bias uncertainty is attributed to possible error associated with the determination of material optical properties, thicknesses, thermocouple readings, and camera temperature readings. An additional uncertainty relating to the approximation of heat losses was also present, but considered as a separate source of bias error. The bias uncertainty in measured heat flux was calculated to be $2.3 \mathrm{~kW} / \mathrm{m}^{2}$ over the range of applied tube heat fluxes in this work. Bias uncertainty in the heat transfer coefficient varied linearly with applied heat flux, ranging from $0.30 \mathrm{~kW} / \mathrm{m}^{2} \mathrm{~K}$ at q" $=2.0 \mathrm{~kW} / \mathrm{m}^{2}$ to $0.73 \mathrm{~kW} / \mathrm{m}^{2} \mathrm{~K}$ at q" $=11.7 \mathrm{~kW} / \mathrm{m}^{2}$. The values of the bias uncertainty were the same order of magnitude of the measurements themselves in most cases, but their effect can be partially negated by an offsetting procedure due to the fact that the error 
sources are fixed values or measurements from set calibration curves (with the exception of heat loss calculations). Therefore, if the measured heat flux for single-phase flow observed just ahead of the bubble is subtracted from the heat flux measured when the bubble is present, then the heat flux enhancement due to the presence of the bubble can be defined as $q^{\prime \prime}{ }_{\text {enh }}=\left(q^{\prime \prime}+\left(\delta q^{\prime \prime}\right)_{\text {bias }}\right)-\left(q^{\prime \prime}{ }_{S P}+\left(\delta q^{\prime \prime}{ }_{S P}\right)_{\text {bias }}\right) \pm\left(\delta q^{\prime \prime}\right)_{\text {heat loss }}=q^{\prime \prime}-q^{\prime \prime}{ }_{S P}+\left(\delta q^{\prime \prime}-\right.$ $\left.\delta q^{\prime \prime}{ }_{S P}\right)_{\text {bias }} \pm\left(\delta q^{\prime \prime}\right)_{\text {heat loss }}$. If the bias uncertainty is same for both measurements, the heat flux enhancement reduces to $q^{\prime \prime}{ }_{\text {enh }}=q^{\prime \prime}-q^{\prime \prime}{ }_{S P} \pm\left(\delta q^{\prime \prime}\right)_{\text {heat loss }}$. If the bias uncertainties are unequal, the difference must be incorporated into the overall uncertainty by propagating the errors.

The random uncertainty was calculated by means of propagation of error for averaged measurements proposed by Taylor [35] in which the averaged uncertainty decreases with respect to the individual measurement uncertainty by a factor of $1 / \sqrt{N}$, where $N$ is the number of averaged measurements. Each temporally averaged bubble heat transfer profile, a discussion of which will follow later, is considered an individual measurement whose random uncertainty is characterized by $\delta_{r}=\frac{\sigma_{r} t}{\sqrt{N}}$, where $\sigma_{r}$ is the standard deviation of the heat flux or heat transfer coefficient about the mean value at each location along the bubble and $t$ is the parameter associated with measurements which are assumed to follow the Student's $t$ distribution. Hereafter, error bars presented in heat transfer figures denote the random uncertainty associated with the particular measurement as well as the uncertainty remaining from bias offsetting of measurements.

\section{RESULTS AND DISCUSSION}

Data was collected in the laboratory and during a parabolic flight campaign which consisted of 164 parabolic maneuvers. Each parabola provided approximately 20 seconds of microgravity $( \pm 0.01 g)$ and 20 seconds of hypergravity $(1.8 g \pm 0.1 g)$ where $g$ is gravitational acceleration. Background liquid velocities within the test section were of the range $35 \mathrm{~mm} / \mathrm{s} \leq U_{l} \leq 140 \mathrm{~mm} / \mathrm{s}$

$\left(50 \mathrm{~kg} / \mathrm{m}^{2} \mathrm{~s} \leq G \leq 200 \mathrm{~kg} / \mathrm{m}^{2} \mathrm{~s}\right)$, which correspond to liquid Reynolds numbers $\left(R e_{l}=\frac{G D}{\mu_{l}}\right)$ between 790 and 3090. The variation of these parameters allowed for heat transfer measurements 
and flow visualization to be obtained at $0.49<B o<87$, thereby spanning both capillary and Taylor bubble regimes. A summary of the test conditions is given in Table 2.

Table 2: Summary of data parameters.

\begin{tabular}{ccccccc}
$\boldsymbol{U}_{\boldsymbol{d}}(\mathbf{m m} / \mathbf{s})$ & $\boldsymbol{G}\left(\mathbf{k g} / \mathbf{m}^{2} \mathbf{s}\right)$ & $\boldsymbol{R e}_{\boldsymbol{l}}$ & $\boldsymbol{a} / \boldsymbol{g}$ & $\boldsymbol{B o}$ & $\boldsymbol{U}_{f}(\mathbf{m m} / \mathbf{s})$ & $\boldsymbol{U}_{\boldsymbol{b}}(\mathbf{m m} / \mathbf{s})$ \\
\hline 20 & 100 & 1580 & 0.01 & 0.48 & - & 90 \\
41 & 200 & 3090 & 0.01 & 0.48 & - & 180 \\
94 & 200 & 3090 & 0.34 & 16.5 & - & 235 \\
105 & 50 & 790 & 1 & 48.4 & 851 & 142 \\
124 & 100 & 1580 & 1 & 48.4 & 1030 & 196 \\
144 & 50 & 790 & 1.8 & 87.2 & 1151 & 180 \\
163 & 200 & 3090 & 1 & 48.4 & 1402 & 305 \\
173 & 100 & 1580 & 1.8 & 87.2 & 1389 & 241 \\
208 & 200 & 3090 & 1.8 & 87.2 & 1754 & 349
\end{tabular}

\subsection{Bubble dynamics}

Elongated bubbles released from the bubble generation section rose into the silicon test section where the heat transfer was measured. High-speed video was then obtained as the bubble passed through the adiabatic glass section. The bubble velocity was determined by tracking the bubble nose as a function of time. A series of 5-20 bubbles were analyzed and averaged to obtain the bubble velocity at each liquid velocity for four gravity levels as shown in Figure 5. For a given liquid velocity, the bubble velocity was found to increase with increasing gravity due to the larger buoyancy force acting on the bubble. Linear curves were fit to the velocity measurements at each gravity level to compare the data with correlations of the form proposed by Nicklin et al. [3]. Results for $U_{b, 0}$ are compared to the predictions of White and Beardmore [5], Rattner and Garimella [7], Brown [6], and Viana et al. [1] in Table 3. Very good agreement is seen with Brown, Viana et al., and White and Beardmore for normal gravity conditions (1g), while the correlations tend to slightly under-predict $U_{b, 0}$ at hypergravity. As expected, $U_{b, 0}$ was very small under microgravity $( \pm 0.01 \mathrm{~g})$ conditions due to the small residual buoyancy force. Similar observations were made by Colin et al. [36] for Taylor flow in microgravity. 


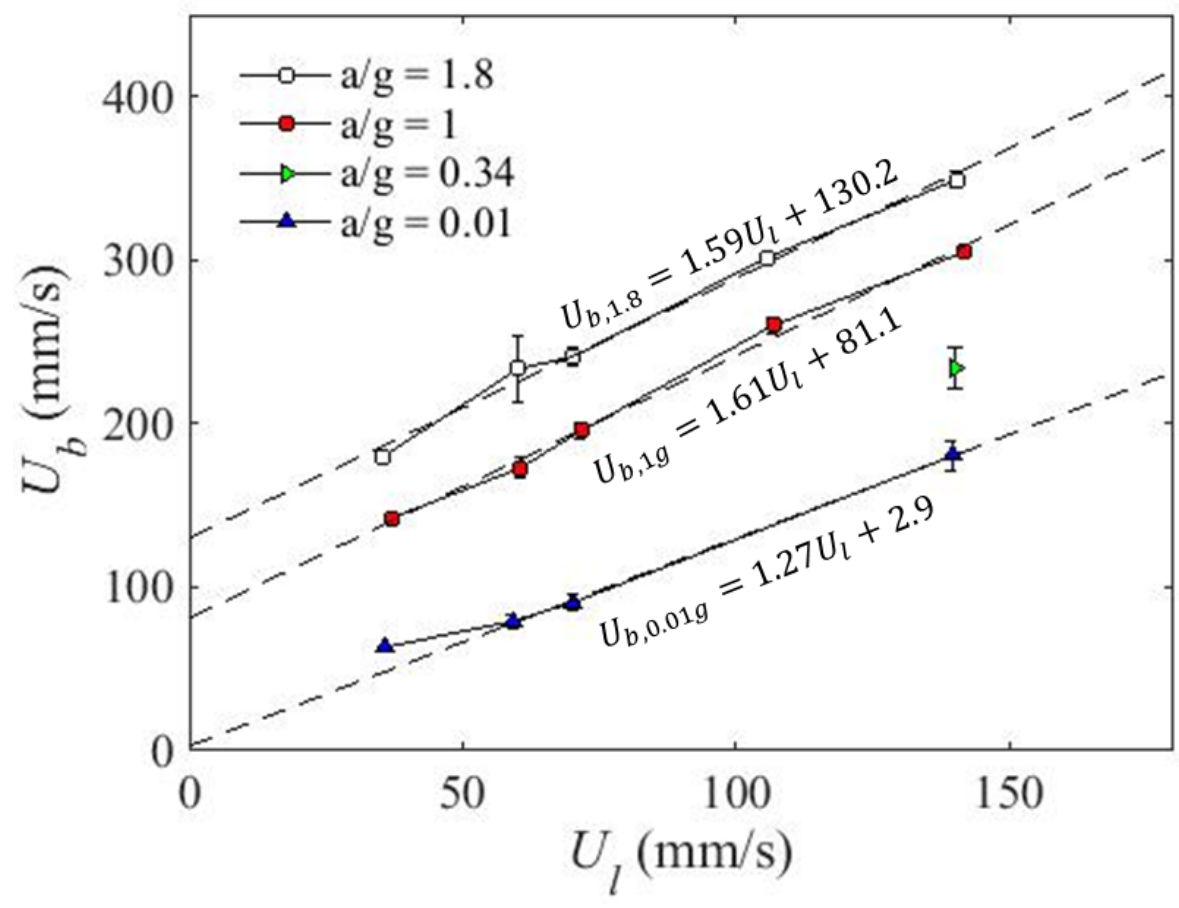

Figure 5: Bubble velocity as a function of liquid velocity for gravitational accelerations tested.

Table 3: Comparison of measured $\mathrm{U}_{\mathrm{b}, 0}$ to those calculated from published correlations.

\begin{tabular}{lccccc} 
a/g & $\begin{array}{c}\text { Present } \\
\text { Study }\end{array}$ & $\begin{array}{c}\text { White and } \\
\text { Beardmore [5] }\end{array}$ & Brown [6] & Viana et al. [1] & $\begin{array}{c}\text { Rattner and } \\
\text { Garimella [7] }\end{array}$ \\
\hline 0.01 & $2.9 \pm 5.8$ & 0.0 & - & - & 0.0 \\
1 & $81.1 \pm 4.1$ & 79.6 & 82.0 & 82.4 & 72.4 \\
1.8 & $130.2 \pm 8.8$ & 112.3 & 110.4 & 110.6 & 108.8
\end{tabular}

Calculated values for $C$ at $1 \mathrm{~g}$ and $1.8 \mathrm{~g}(B o=49$ and 87$)$ were found to be consistent with the $1 \mathrm{~g}$ results of Scammell and Kim [21] which fell between the classical bounds of 2 and 1.2 for the laminar and turbulent liquid flow regimes, respectively. Under microgravity conditions $(B o=0.49), C$ was measured to be smaller than at normal and hypergravity. The variation is likely due to a transition from Taylor flow to capillary flow given that the bubble $B o$ in microgravity lies below the critical values suggested by previous authors [5,8,22-24]. Visual evidence of a transition is provided in Figure 6 where high-speed images of bubbles rising at various gravity 
levels and bubble drift velocities are shown. Under microgravity conditions (Figure 6a,b), the bubbles exhibited rounded tail profiles whose radius of curvature increased with drift velocity. Stationary, small amplitude interfacial waves were observed at $U_{d}=20 \mathrm{~mm} / \mathrm{s}$ (Figure 6a). When the drift velocity increased to $41 \mathrm{~mm} / \mathrm{s}$ (Figure 6b), the waves became more frequent, larger in amplitude, and oscillated in the streamwise direction. Above $U_{d}=94 \mathrm{~mm} / \mathrm{s}$ (Figure $6 \mathrm{c}-\mathrm{e}$ ), the Taylor bubbles possessed flat tails that wobbled as the bubbles rose. The magnitude of the wobble increased with bubble length as was seen by Polonsky et al. [37] and Scammell and Kim [21].

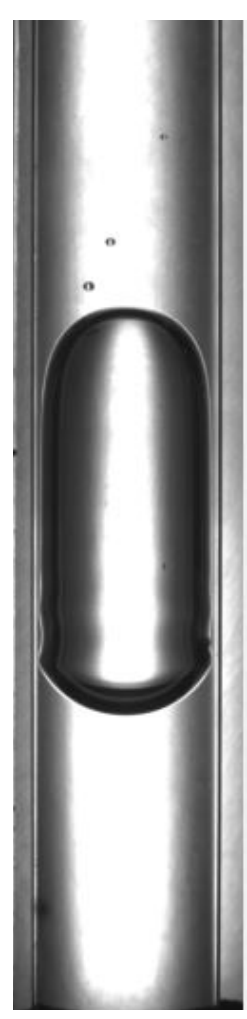

a)

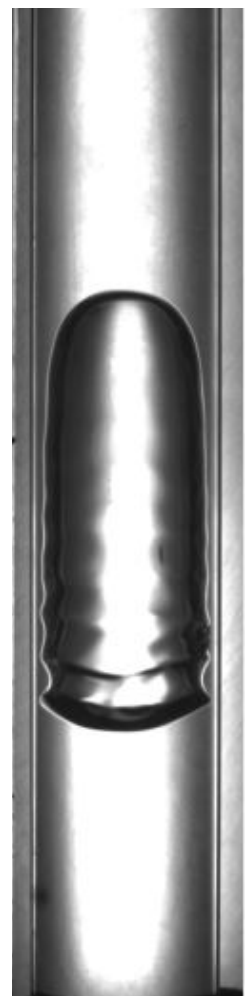

b)

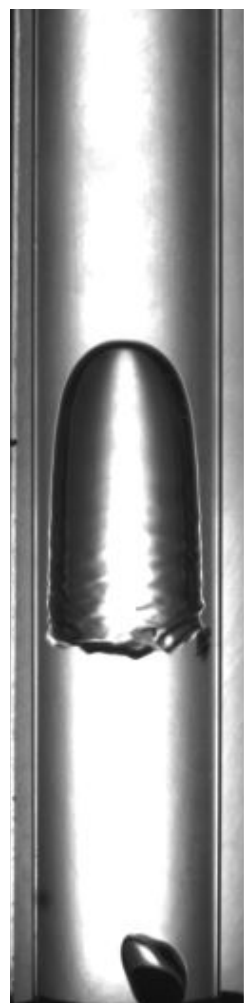

c)

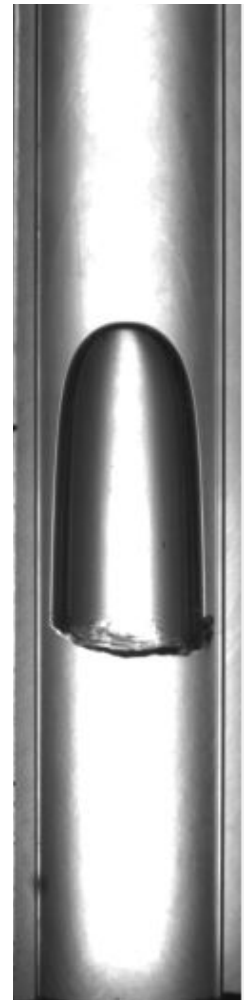

d)

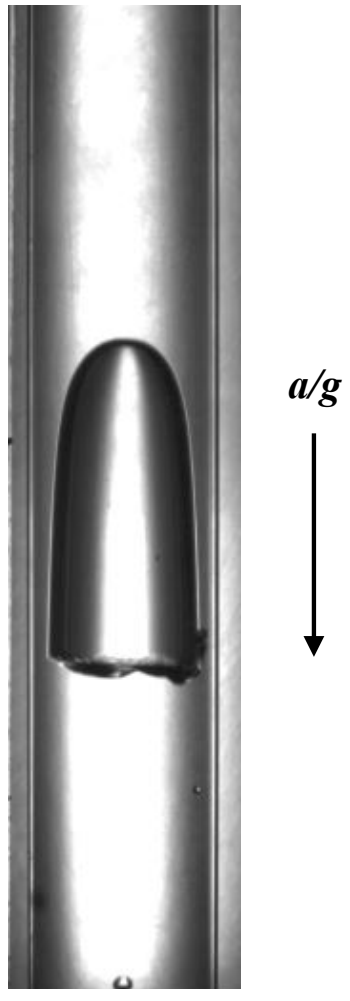

e)

Figure 6: Images of representative bubbles at varying accelerations and drift velocities: a) $\mathrm{a} / \mathrm{g}=0.01, U_{d}$ $=20 \mathrm{~mm} / \mathrm{s}, B o=0.97, L=17 \mathrm{~mm}$; b) $a / g=0.01, U_{d}=41 \mathrm{~mm} / \mathrm{s}, B o=0.97, L=18 \mathrm{~mm}$; c) $a / g=0.34, U_{d}=$ $94 \mathrm{~mm} / \mathrm{s}, B o=16.6, L=13 \mathrm{~mm}$;) $a / g=1, U_{d}=106 \mathrm{~mm} / \mathrm{s}, B o=48.7, L=13 \mathrm{~mm}$; e) $a / g=1.8, U_{d}=214$ $\mathrm{mm} / \mathrm{s}, B o=87.7, L=13 \mathrm{~mm}$.

A more detailed bubble profile analysis was conducted using the open source image processing program ImageJ [38]. The nose profiles of bubbles with drift velocities of $U_{d}=20,41$, 
94, 175, and $214 \mathrm{~mm} / \mathrm{s}$ are compared in Figure 7. The bubbles exhibit narrower, more rounded nose contours with increasing $U_{d}$. The theoretical nose shape suggested by Dumitrescu [39] for a Taylor bubble rising in a stagnant column of fluid without viscosity or surface tension at normal gravity is also included. With a corresponding drift velocity of approximately $81 \mathrm{~mm} / \mathrm{s}$ (the value of $U_{b, 0}$ at $1 \mathrm{~g}$ ), Dumitrescu's profile is seen to fall between the experimental curves for $U_{d}=41$ and $94 \mathrm{~mm} / \mathrm{s}$ near the top of the bubble. As the liquid film develops, the prediction moves within the measured profile for $U_{d}=94 \mathrm{~mm} / \mathrm{s}$ but remains within the uncertainty of the measurements.

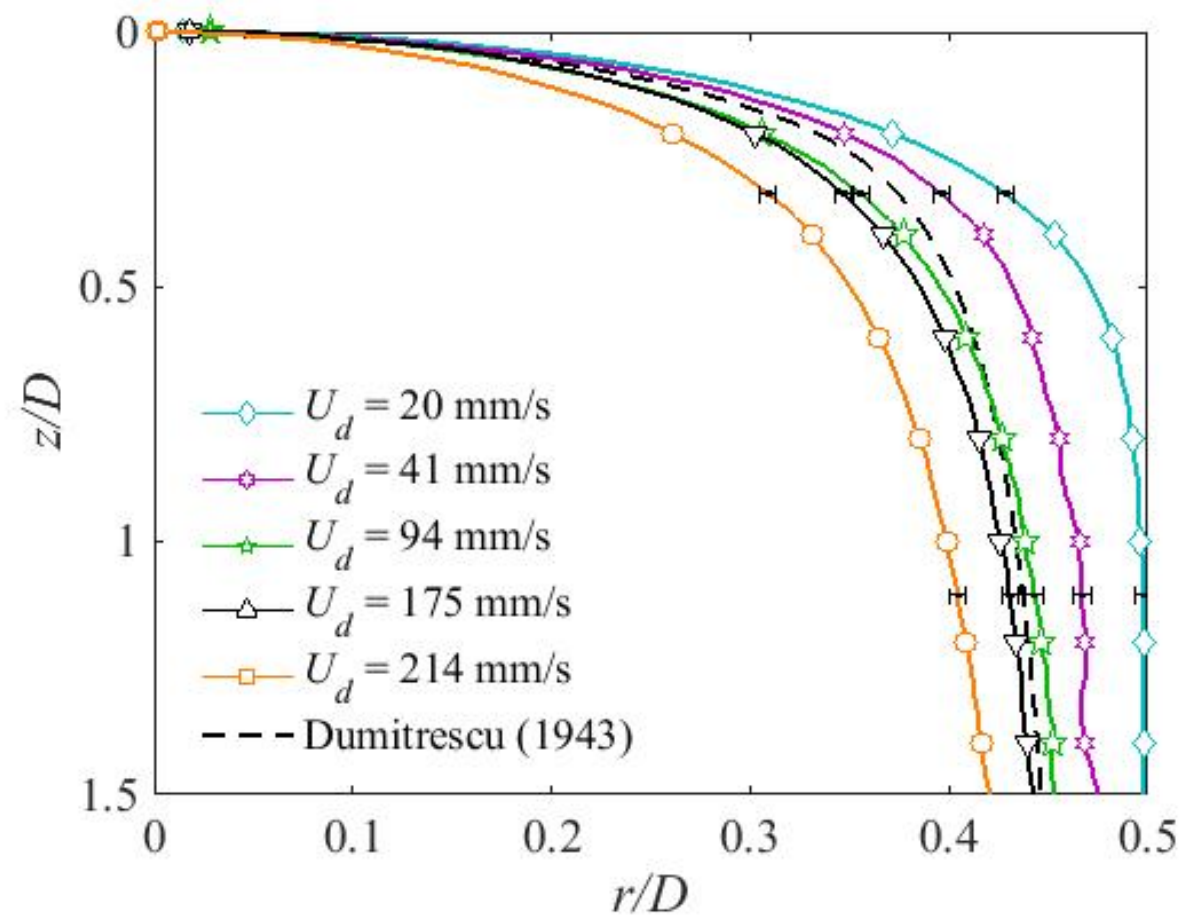

Figure 7: Bubble nose profiles at various drift velocities.

As was mentioned, small waves were observed for capillary bubbles $\left(U_{d}=20\right.$ and $\left.41 \mathrm{~mm} / \mathrm{s}\right)$ at the vapor/liquid interface near the bubble tail. Profiles for two representative bubbles at these drift velocities are shown in Figure 8. The wavelength $(\lambda)$ was found to be slightly dependent upon $U_{d}$ with values of $2.13 \pm 0.01 \mathrm{~mm}$ and $1.79 \pm 0.01 \mathrm{~mm}$ for $U_{d}=20$ and $41 \mathrm{~mm} / \mathrm{s}$, respectively. Ratulowski and Chang [40] numerically predicted the shape of elongated bubbles as a function of the Capillary number $\left(C a_{b}=\frac{\mu U_{b}}{\sigma}\right)$. A series of relations were developed for the characteristic wavelength of capillary waves at the bubble interface. For $\mathrm{Ca}=0.0034\left(U_{d}=20 \mathrm{~mm} / \mathrm{s}\right)$ and 
$\mathrm{Ca}=0.0069\left(U_{d}=41 \mathrm{~mm} / \mathrm{s}\right)$, Ratulowski and Chang predict $\lambda$ to be $2.15 \mathrm{~mm}$ and $2.83 \mathrm{~mm}$, quite similar to the current experimental results. Edvinsson and Irandoust [41] compared their numerical results for $\lambda$ to those of Ratulowski and Chang, and found that $\lambda / D$ was overpredicted by the original results for $\mathrm{Ca}>0.005$. Edvinsson and Irandoust's results can be applied to the current study yielding a wavelength of $2.41 \mathrm{~mm}$ for bubbles with $U_{d}=41 \mathrm{~mm} / \mathrm{s}$, which is somewhat closer to the current measurement of $1.79 \mathrm{~mm}$ than from Ratulowski and Chang.
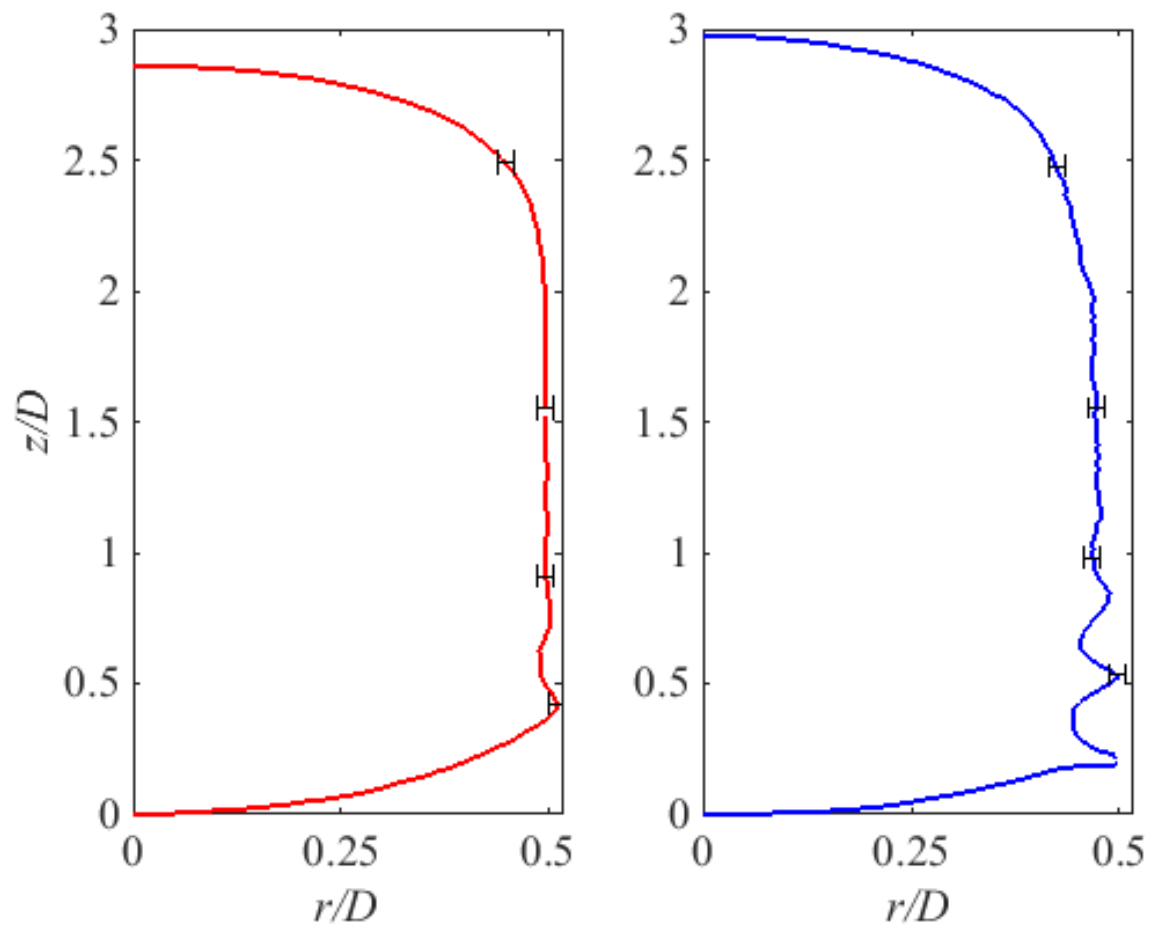

Figure 8: Bubble shape profiles for $U_{d}=20 \mathrm{~mm} / \mathrm{s}$ (left) and $U_{d}=41 \mathrm{~mm} / \mathrm{s}$ (right) under microgravity conditions.

The liquid film thickness for Taylor bubbles were obtained using either the Keyence sensor or high-speed image analysis as described previously. Figure 9 compares data collected at $a / g=1$ (Keyence sensor) and $a / g=1.8$ (image analysis) with their respective uncertainties labeled on several points. The tail film thickness for bubbles with $U_{d}=105,124,163,173$, and $208 \mathrm{~mm} / \mathrm{s}$ are plotted as a function of the bubble length. As expected, $\delta$ decreases with bubble length until approximately $L_{b} / D=4$ where the thickness reaches the fully developed value. The data is compared to predictions by Brown [6] for fully developed film thickness $\left(\delta_{B}\right)$ by two means. First, $\delta_{B}$ was calculated for each value of $U_{d}$ using the measured $U_{b}, U_{l}$, and an assumed 
acceleration of $1 g$. This range of $\delta_{B}$ is illustrated by a blue area in Figure 9. Also shown is the prediction for $U_{d}=163 \mathrm{~mm} / \mathrm{s}$ and $a / g=1$, which agrees well with the measurements at these conditions. The calculations were repeated for $U_{d}=173$ and $208 \mathrm{~mm} / \mathrm{s}$ at hypergravity, with the results shown by the red area and dash-dot line $\left(U_{d}=173 \mathrm{~mm} / \mathrm{s}\right)$. It is clear that the relation by Brown over-predicts the film thickness for higher gravity levels. In fact, the measurements at $a / g=1.8$ vary little from those collected at $a / g=1$. Using the correlation of Llewellin et al. [12] for fully developed film thickness of Taylor bubbles in stagnant flow, $\delta$ was calculated to be $271 \mu \mathrm{m}$ for $a / g=1$ and $264 \mu \mathrm{m}$ for $a / g=1.8$. Both of these values fall within the $1 g$ prediction range of Brown and illustrate relatively little change in film thickness with increasing gravity level, which is consistent with the measurements.

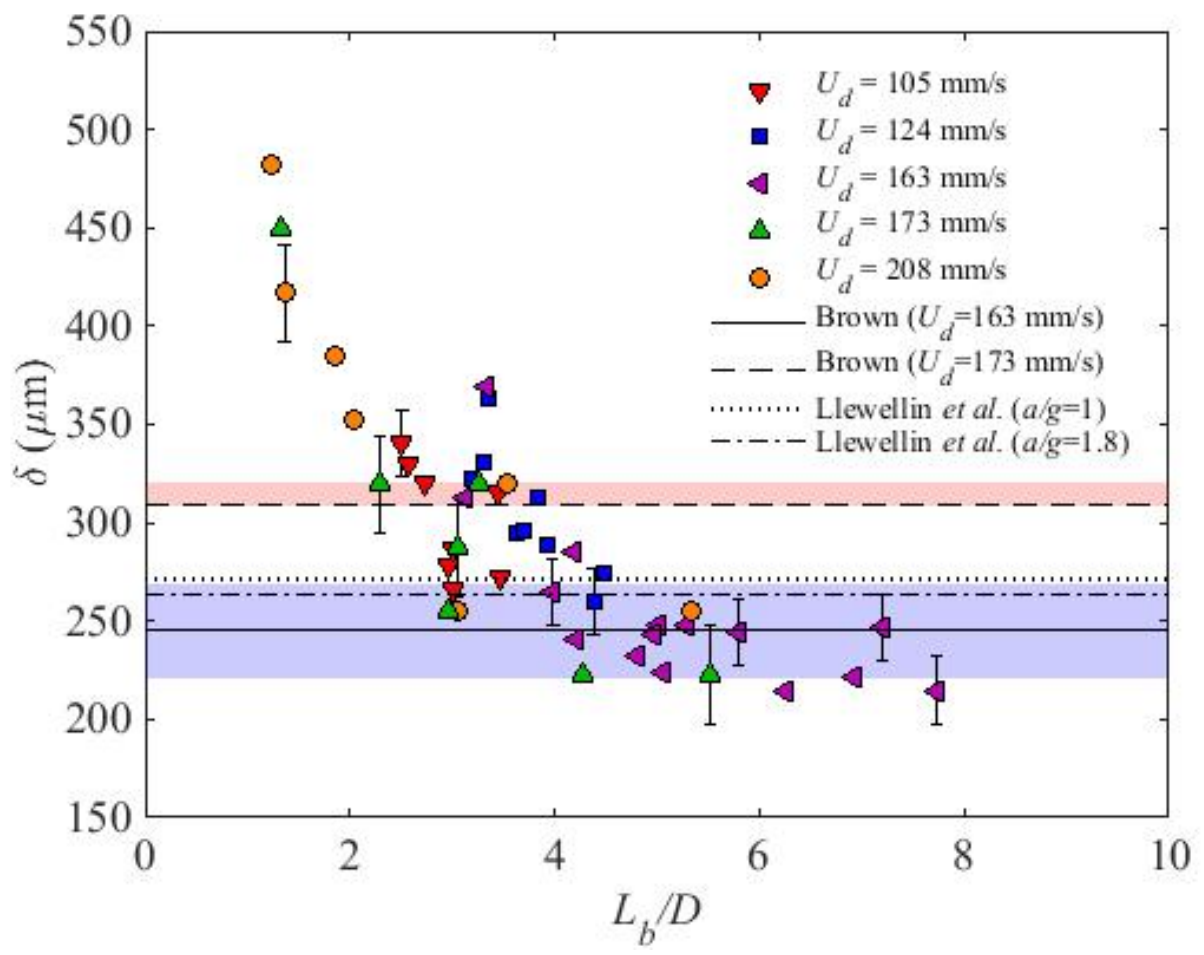

Figure 9: Film thickness at the bubble tail as a function of bubble length at various drift velocities.

\subsection{Heat transfer}

The wall heat transfer was measured for each bubble as it passed through the silicon test section where steady-state, thermally developing flow was established to ensure reproducible boundary 
conditions. Measurements were offset by the single-phase heat transfer observed just before the bubble enters the test section. This means of characterization was chosen to emphasize the effect of heat transfer enhancement created by the bubble passage, rather than the effect of the background flow conditions. Two heat transfer coefficient profiles $\left(h-h_{S P}\right)$ obtained at a Reynolds number of $R e_{l}=1580$ ( $a / g=0.01$ and 1) are plotted in Figure 10 as a function of axial location along the bubble, where $z / D=0$ indicates the bubble tail. The profiles shown are an average of the instantaneous profiles measured in the frame of reference of a viewer moving with the bubble tail. For both gravity levels, the heat transfer coefficient ahead of the bubble corresponds to single-phase flow. As the liquid thins during bubble passage, a slight enhancement to the heat transfer occurs due to increased conduction and convection. Waves in the liquid-vapor interface create oscillations in the capillary bubble heat transfer (Figure 10a) as the fluid accelerates and decelerates within the film. At the capillary bubble tail $(\sim z / D=0)$, a significant decline in the heat transfer enhancement was observed and may be attributed to a local thickening of the thermal boundary layer caused by a negative radial velocity in this region. Magnini et al. [27] also observed these flow characteristics in their numerical simulations of capillary flow within a microchannel. No capillary oscillations (shape or heat transfer) are seen in the Taylor bubble profile (Figure 10b) where the interface remains smooth. At the tail of the Taylor bubble, vortices are generated when the downward moving film plunges into the trailing liquid slug, inducing turbulence and a large spike in heat transfer. Vortices were not observed in the wake of the capillary bubble and the heat transfer coefficient was seen to be similar to singlephase flow. Representative IR flow visualization images of the two bubbles are shown in Figure 11. The wake (to the left of the image) of the capillary bubble was seen to maintain an essentially uniform temperature, suggesting that the near-wake region remained laminar and no mixing occurred. Just behind the Taylor bubble, however, clear temperature fluctuations (vortices) can be seen, indicating mixing of warmer and cooler fluid. 


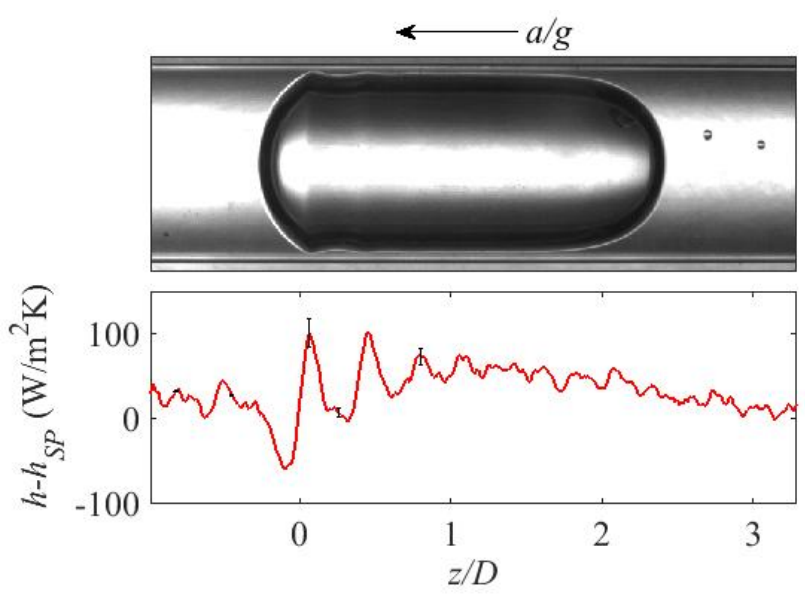

a)

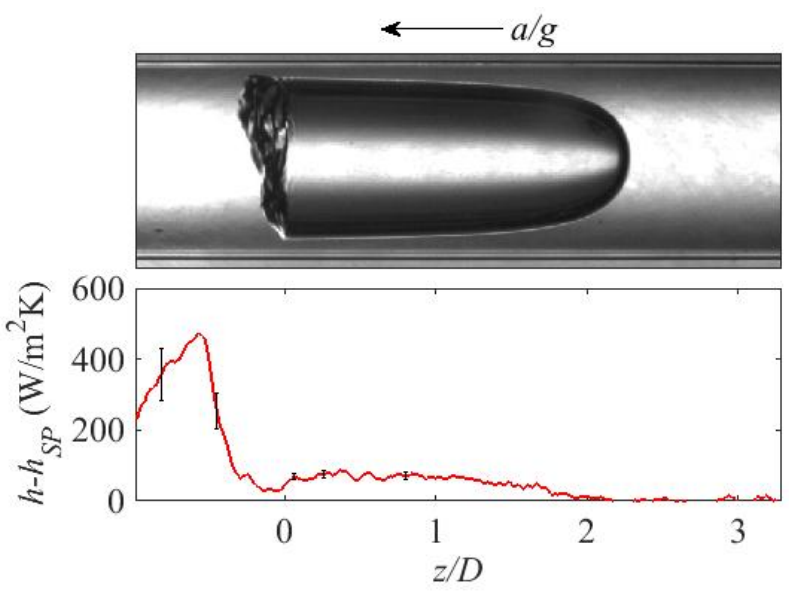

b)

Figure 10: Representative heat transfer coefficient profiles: a) capillary bubble $\left(U_{d}=20 \mathrm{~mm} / \mathrm{s}, U_{l}=70\right.$ $\left.\left.\mathrm{mm} / \mathrm{s}, L_{b}=16 \mathrm{~mm}, a / g=0.01, q "=800 \mathrm{~W} / \mathrm{m}^{2}, h_{S P}=101 \mathrm{~W} / \mathrm{m}^{2} \mathrm{~K}\right), \mathrm{b}\right)$ Taylor bubble $\left(U_{d}=124 \mathrm{~mm} / \mathrm{s}, U_{l}=70\right.$ $\left.\mathrm{mm} / \mathrm{s}, L_{b}=14 \mathrm{~mm}, a / g=1, q "=1440 \mathrm{~W} / \mathrm{m}^{2}, h_{S P}=148 \mathrm{~W} / \mathrm{m}^{2} \mathrm{~K}\right)$.

a)
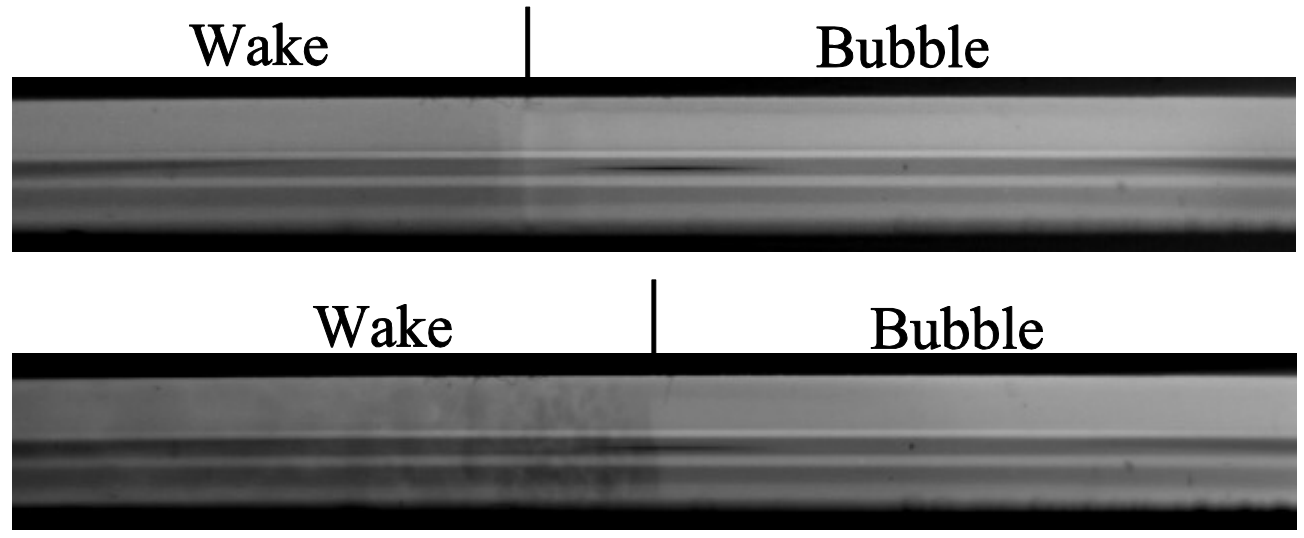

Figure 11: Representative IR images of the near wake region for bubbles presented in Figure 10: a) capillary bubble and b) Taylor bubble.

For Taylor bubbles, the bubble length was found to have little effect on the shape and magnitude of the wake heat transfer enhancement as shown in Figure 12. The enhancement due to five bubbles of different lengths at $U_{d}=163 \mathrm{~mm} / \mathrm{s}$ differed by an amount similar to the uncertainty in the measurements and no visible trend in the profiles was seen. If it is assumed that the relative velocity between the liquid film and trailing liquid slug is the parameter that dictates wake heat transfer, then this result is not surprising. As was seen in Figure 9, the liquid film thickness is essentially invariant for $L_{b} / D>4$. The bubbles presented in Figure 12 were very 
near or above this threshold, suggesting that their film velocities and subsequently wake heat transfer should also be similar. Babin et al. [20], on the contrary, found that the magnitude and length of the wake enhancement increased with bubble length for both laminar and turbulent flows. They attributed the trends to two factors. First, the higher film velocities associated with longer bubbles resulted in vortices that persisted farther downstream. Second, the increased wobbling of the tail for longer bubbles may have resulted in increased wake enhancement.

An additional explanation arises if the bubble development length is considered. The bubble length required for a fully developed liquid film was calculated for the conditions of Babin et al. [20] to be $L_{b} / D \approx 22$ using Campos and Carvalho [9] where the film thickness was approximated using Brown [6]. The bubble lengths considered by Babin et al. ranged from $L_{b} / D=2-10$, significantly shorter than the $L_{b} / D$ for fully developed flow. It is possible that the enhancement in wake heat transfer observed with increasing bubble length is the result of higher film velocities. As the bubbles in the current experiment were essentially fully developed, little variation in the wake heat transfer profiles are seen for the results. 


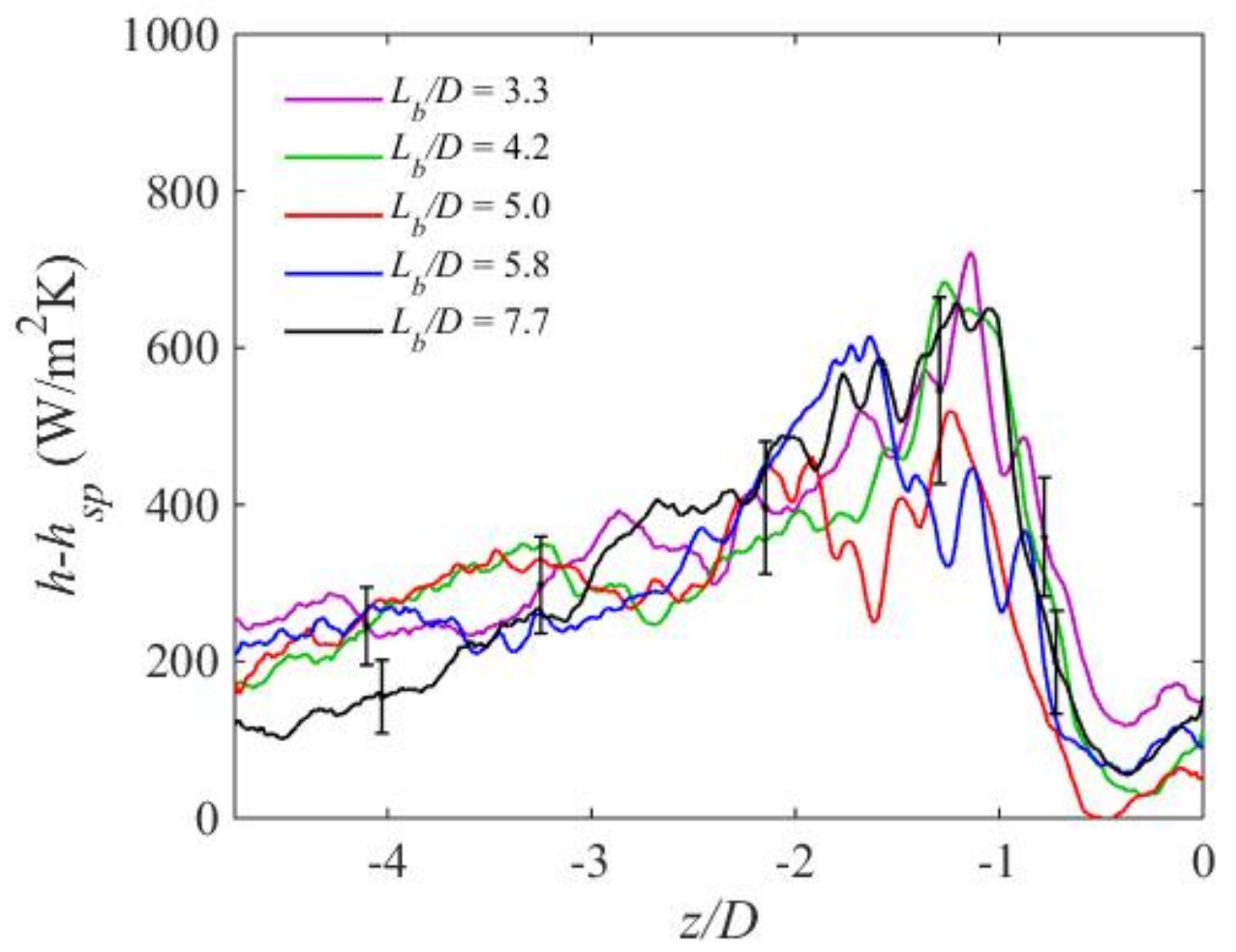

Figure 12: Scaled heat transfer coefficient as a function of distance behind the bubble tail for bubble drift velocity $U_{d}=163 \mathrm{~mm} / \mathrm{s}$, tube heat flux of $q^{\prime \prime}=1.0 \mathrm{~kW} / \mathrm{m}^{2}$, average single-phase heat transfer coefficient $h_{S P}=192 \mathrm{~W} / \mathrm{m}^{2} \mathrm{~K}$, and bubble lengths $L_{b} / D=3.2,4.2,5,5.8$, and 7.7 .

A 2-D plot of the temporal and spatial wake heat transfer can be used to infer the local effect of vortices generated by Taylor bubbles. An example of such a plot and the wake characteristics are shown in Figure 13. The resolution of the data was limited by the spatial $(114 \mu \mathrm{m}, 0.02 D)$ and temporal (4 ms) resolution of the camera, thus creating the pattern of colored rectangles. Streaks of elevated heat transfer (labeled by black lines) were likely caused by the replacement of the plunging jet with cooler liquid when the jet separates from the tube wall. The distance behind the tail at which the streaks appear (the "penetration length", $L_{p}$ ) is seen to vary with respect to time, a behavior that can be attributed to two factors as illustrated in Figure 14. First, the location of the bubble tail $(z / D=0)$ is determined from the average bubble velocity and does not account for wobbling of the tail. As a result, the actual location of the tail at each time may be slightly ahead or behind the average position, leading to a variation in the perceived penetration length. The second factor to be considered is the instability inherent in the plunging jet which would likely lead to oscillations in $L_{p}$ even if the tail position was fixed. 


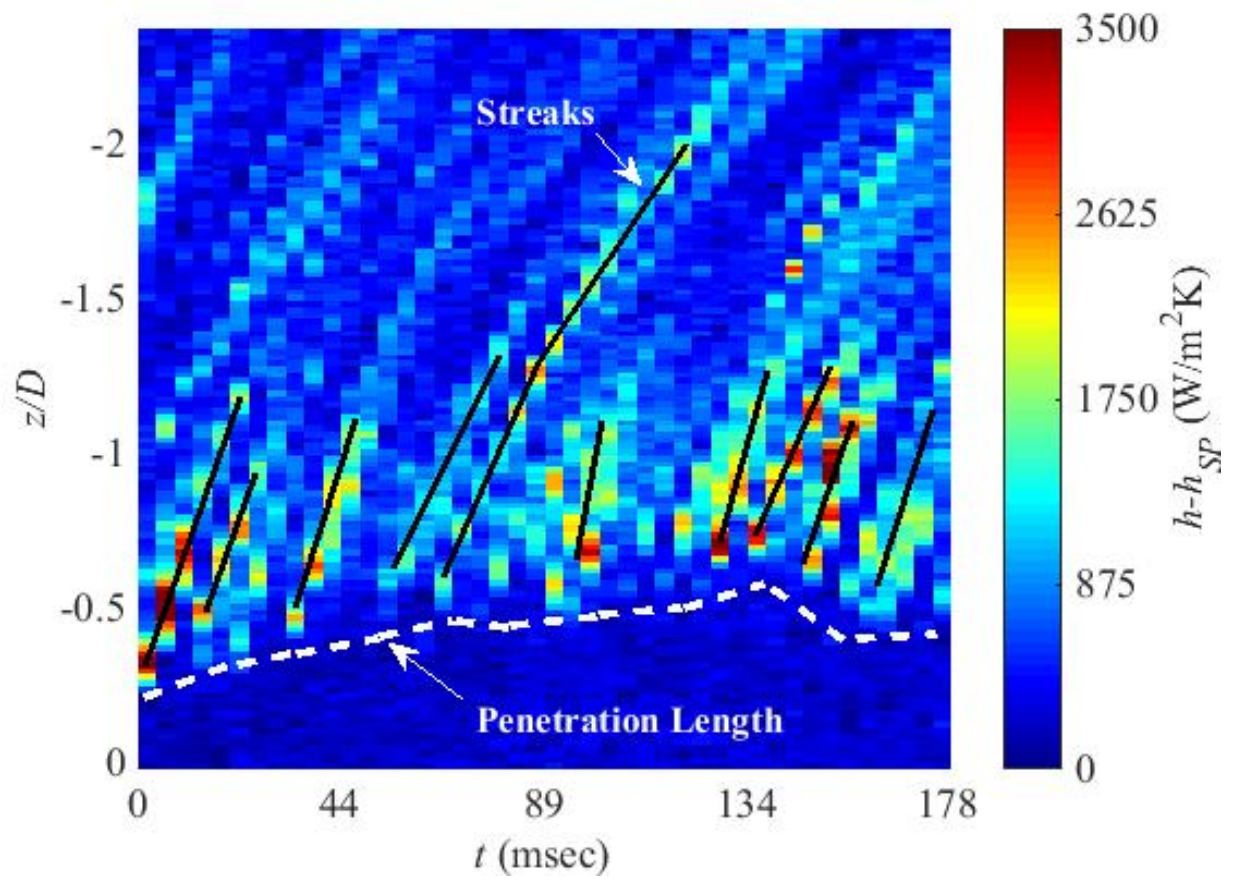

Figure 13: Representative wake heat transfer signature characteristics identification.

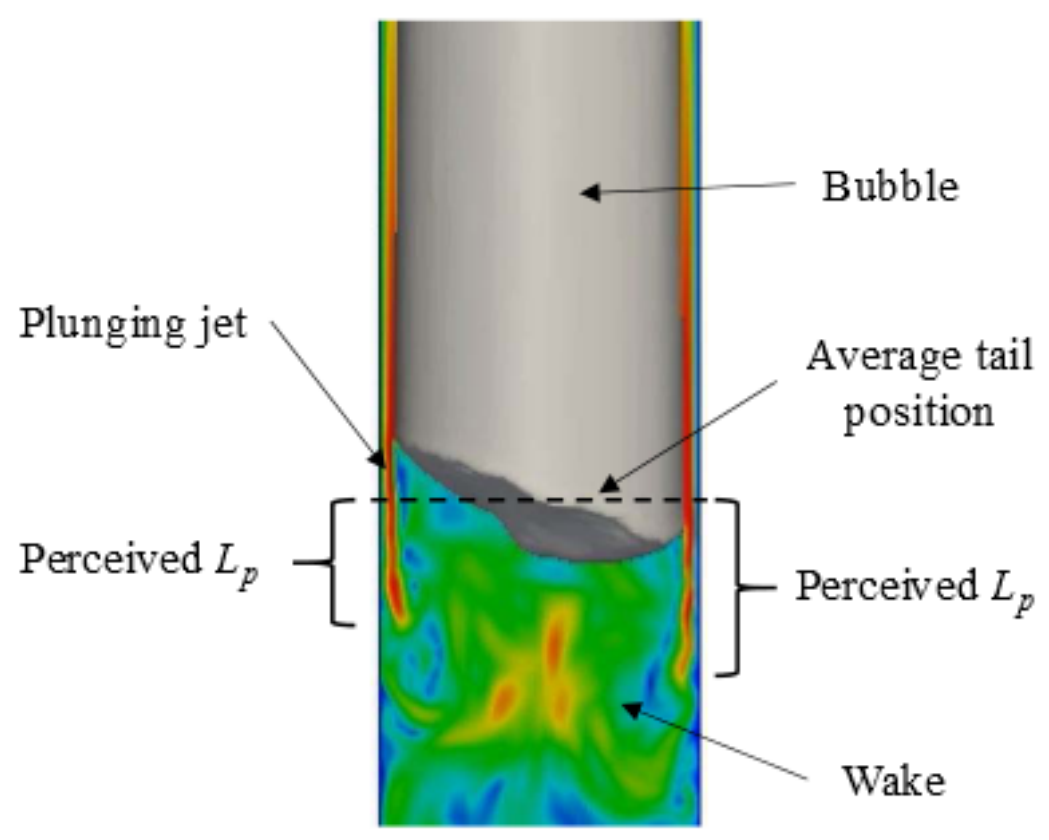

Figure 14: Schematic showing high velocity wall jets (red) plunging into the wake. Separation of the jet occurs at the penetration length. 
Heat transfer coefficient contours for flows with $R e_{l}=790,1570$, and 3090 at $a / g=1$ and $a / g=1.8$ are shown in the left images of Figure 15 and Figure 16, respectively. Plots on the right illustrate the heat transfer coefficient profile at four time steps. These individual profiles confirm the variation in $L_{p}$, which is seen to range from 0.5-1.5 diameters for bubbles observed at $a / g=1$ and 0.7-2.0 diameters for $a / g=1.8$. Similar results were obtained by Kawaji et al. [15] for air bubbles rising in stagnant kerosene, where $L_{p}$ ranged from 0.85-1.4 diameters depending on the bubble length. Shemer et al. [16,42], while not explicitly describing the penetration length, found that the highest time-averaged radial velocity in the wake occurred at approximately 1 diameter from the tail. This is consistent with the current observations that the streaks are initiated at the point where the hot wall jet separates from the wall and is replaced by cooler liquid from the tube center. 

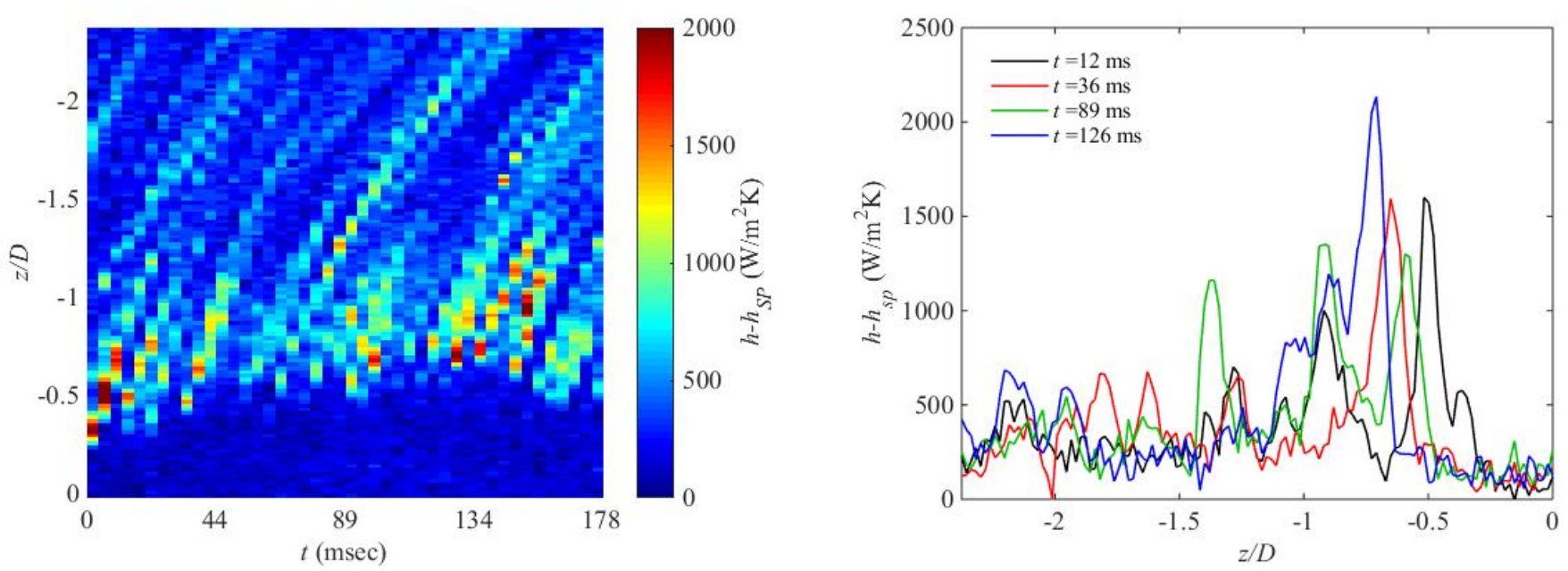

a) $U_{d}=105 \mathrm{~mm} / \mathrm{s}, q "=1400 \mathrm{~W} / \mathrm{m}^{2}, h_{S P}=130 \mathrm{~W} / \mathrm{m}^{2} \mathrm{~K}$
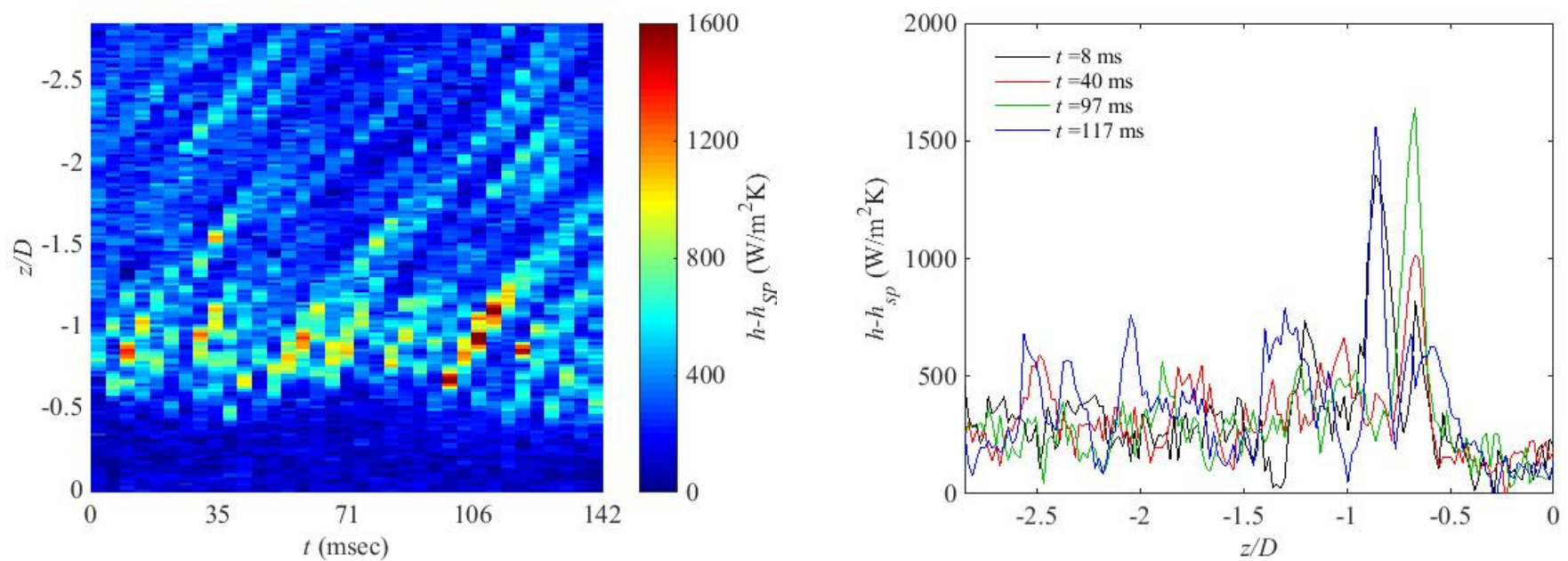

b) $U_{d}=124 \mathrm{~mm} / \mathrm{s}, q^{\prime \prime}=1470 \mathrm{~W} / \mathrm{m}^{2}, h_{S P}=148 \mathrm{~W} / \mathrm{m}^{2} \mathrm{~K}$
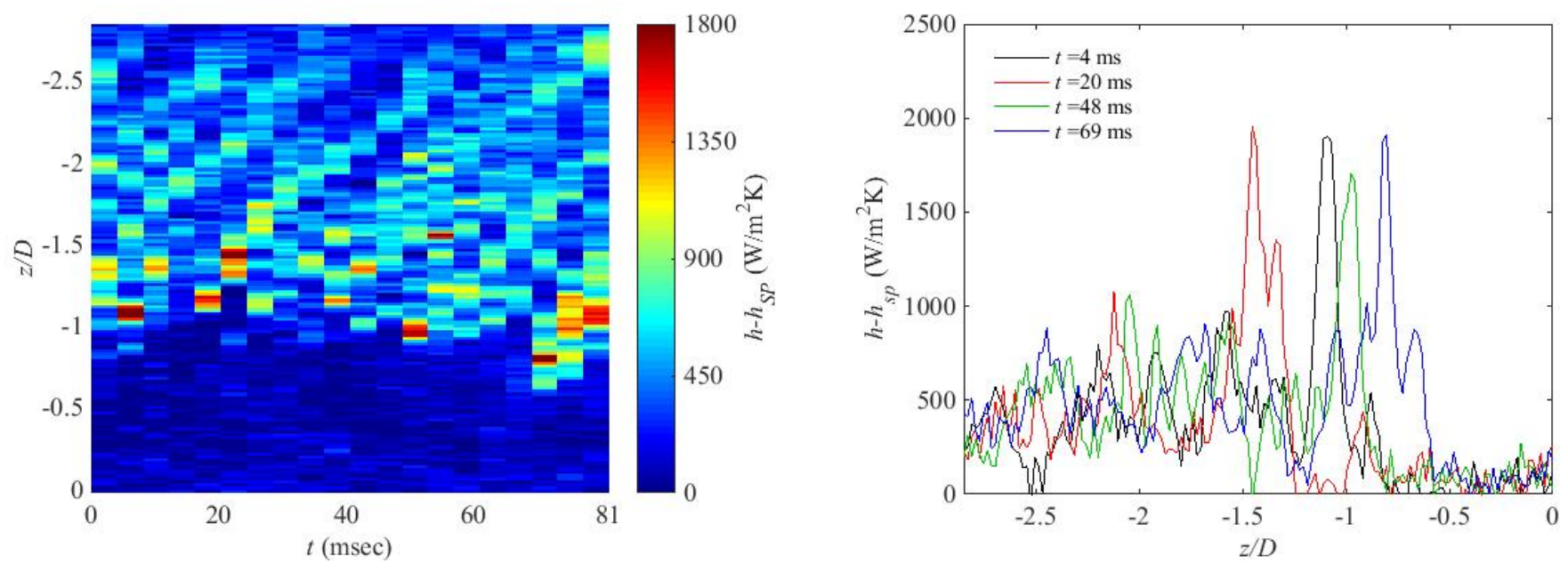

c) $U_{d}=163 \mathrm{~mm} / \mathrm{s}, q^{\prime \prime}=1700 \mathrm{~W} / \mathrm{m}^{2}, h_{S P}=192 \mathrm{~W} / \mathrm{m}^{2} \mathrm{~K}$

Figure 15: Wake heat transfer coefficient contours (left) and representative profiles (right) at $a / g=1$ for various conditions. 

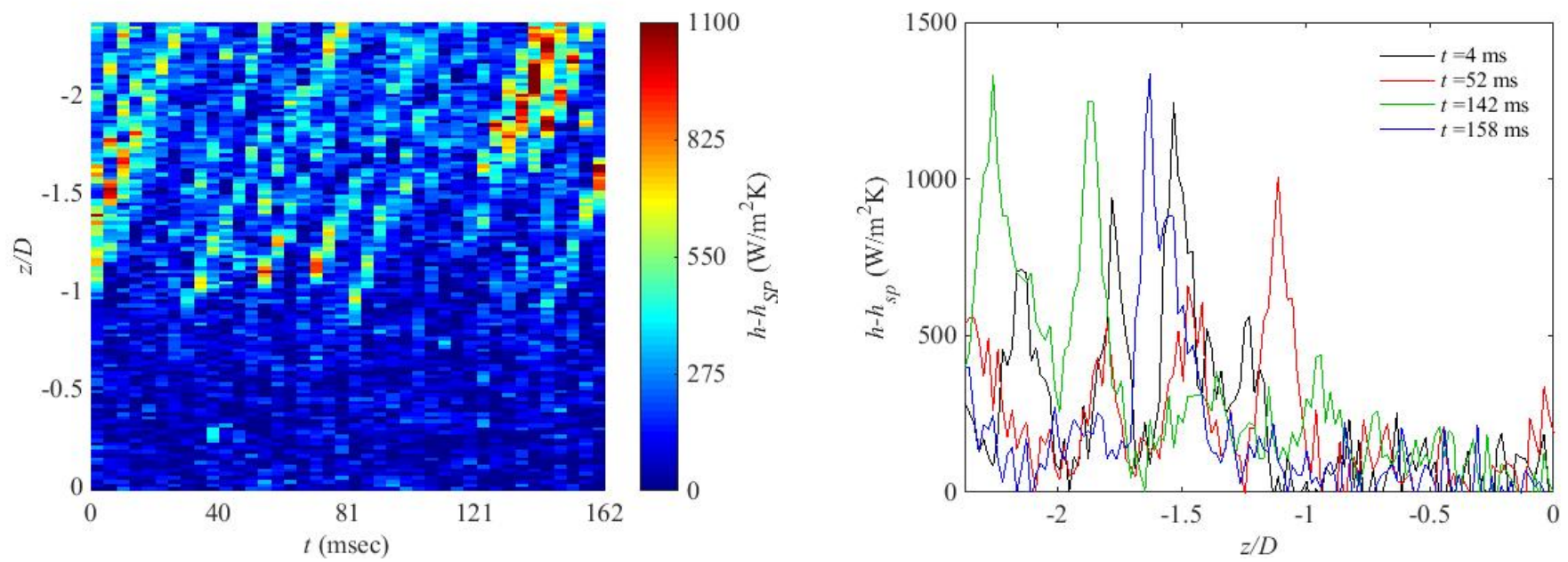

a) $U_{d}=144 \mathrm{~mm} / \mathrm{s}, q^{\prime \prime}=1140 \mathrm{~W} / \mathrm{m}^{2}, h_{S P}=104 \mathrm{~W} / \mathrm{m}^{2} \mathrm{~K}$
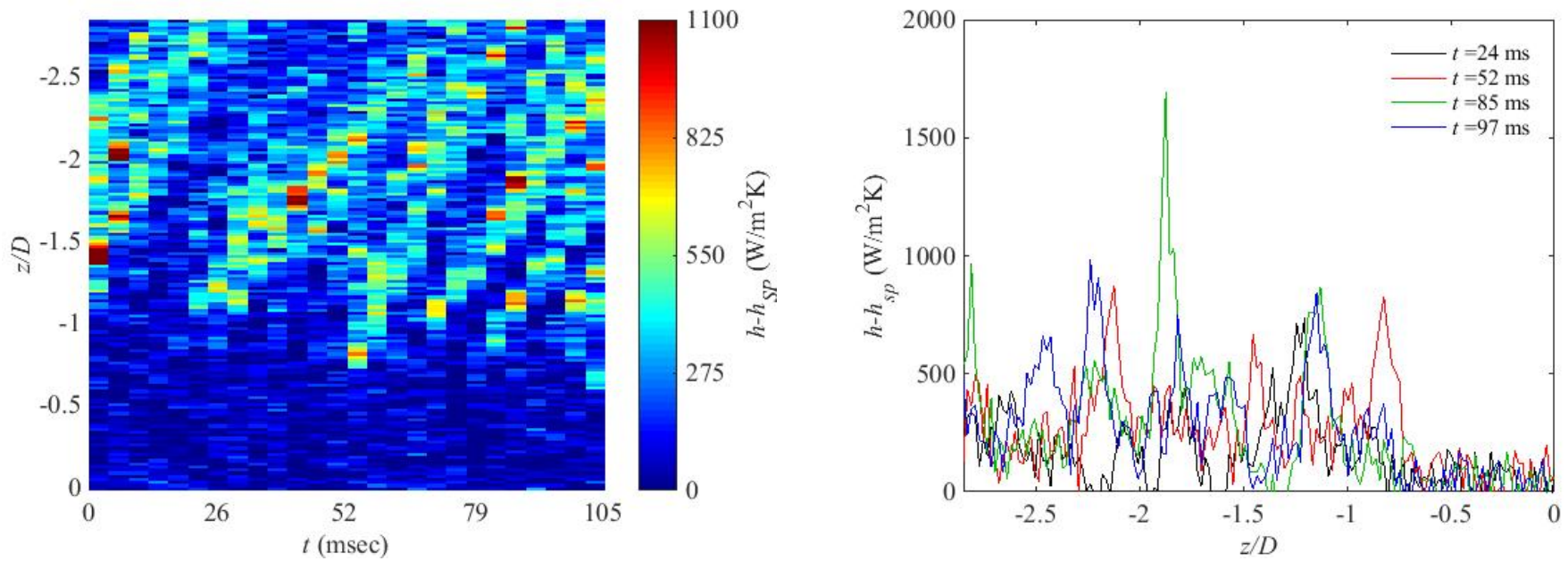

b) $U_{d}=173 \mathrm{~mm} / \mathrm{s}, q^{\prime \prime}=1330 \mathrm{~W} / \mathrm{m}^{2}, h_{S P}=112 \mathrm{~W} / \mathrm{m}^{2} \mathrm{~K}$
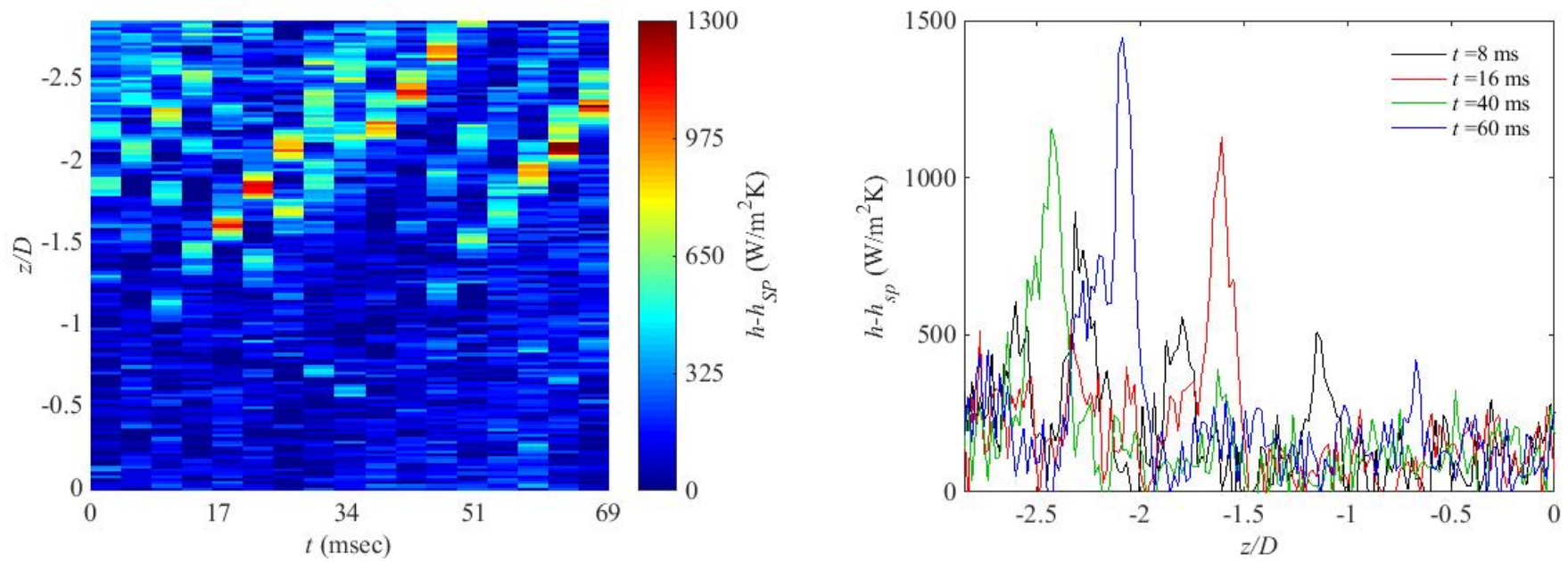

c) $U_{d}=208 \mathrm{~mm} / \mathrm{s}, q^{\prime \prime}=1320 \mathrm{~W} / \mathrm{m}^{2}, h_{S P}=146 \mathrm{~W} / \mathrm{m}^{2} \mathrm{~K}$

Figure 16: Wake heat transfer coefficient contours (left) and representative profiles (right) at $a / g=1.8$ for various conditions. 
The vortex frequency, $f_{v}$, was determined by counting the number of streaks and dividing by the time over which they occurred. At each $U_{p}, f_{v}$ was found by averaging the calculated frequencies for a number of contour plots (between 3 and 13) at each flow condition. Attempts were made at performing Fourier analyses on the streaks. However, due the limited length of the acquired signals (resulting from the limited test section length) and quasi-periodicity of vortex generation, the results were inconclusive. The calculated frequency results should serve as an approximation of the wake turbulence. The frequency increases monotonically with increasing $U_{p}$ as seen in Figure 17, and is independent of gravity level for $a / g=1$ and 1.8. It is possible that the two bubbles observed under Martian gravity $(a / g=0.34)$ fall within a transitional region between capillary and Taylor bubble hydrodynamics and therefore do not align with the higher gravity data. Scammell and Kim [21] found that the vortex frequency remained relatively constant with $U_{d}$, but this conclusion was based on a limited range of $U_{d}$ and a limited number of bubbles. A linear fit to the current $a / g=1$ and 1.8 data, which is based on a much larger dataset that includes a wider range of $U_{d}$ made possible by varying the gravity level, suggests that a critical plunging velocity $\left(U_{p, c r}\right)$ of $127 \mathrm{~mm} / \mathrm{s}$ exists where vortices begin to appear. For this experiment, $U_{p, c r}=127 \mathrm{~mm} / \mathrm{s}$ corresponds to a critical drift velocity of $U_{d, c r}=34 \mathrm{~mm} / \mathrm{s}$ and a critical drift Reynolds number of $R e_{U_{d, c r}}=741$. Pinto et al. [14] proposed $R e_{U_{d, c r}}=525$ as the boundary for incipience of vortex shedding, which compares well with the current result. The frequency can be generalized using the Strouhal number $(S t)$, defined here as $S t=\frac{f_{v} \delta}{\left(U_{p}-U_{p, c r}\right)}$, to be $S t=0.018 \pm 0.003$. 


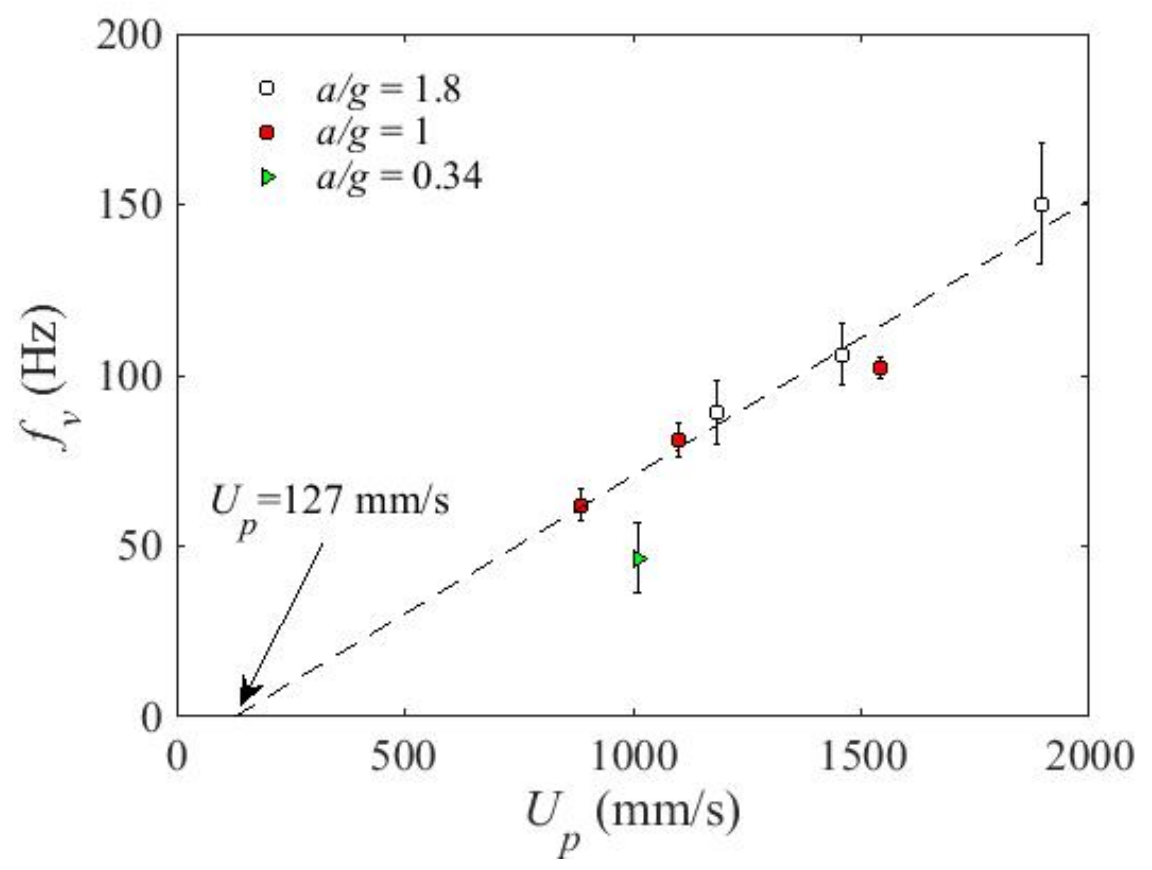

Figure 17: Wake vortex frequency as a function of the relative velocity between the film and bulk liquid velocity for $a / g=1$ and 1.8 .

The velocity with which the vortices moved away from the tail in the bubble reference frame, $U_{v}^{\prime}$, was determined from the streaks in Figure 15 and Figure 16 by measuring their slope in the near-wake region $(-2.5 \leq z / D \leq 0)$. $U_{v}^{\prime}$ was normalized with the wall velocity, $U_{w}^{\prime},\left(U_{w}^{\prime}=U_{b}\right)$ and plotted in Figure 18 as a function of $U_{w}^{\prime}$. It can be seen that the ratio of the vortex velocity to the wall velocity is significantly larger than unity at low wall velocities and linearly decreases with increasing wall velocity to approximately unity. This trend is potentially related to the penetration length at each value of $U_{w}^{\prime}$ and the decay of the film as it moves downstream, but more detailed flow analysis is required for a more thorough explanation. 


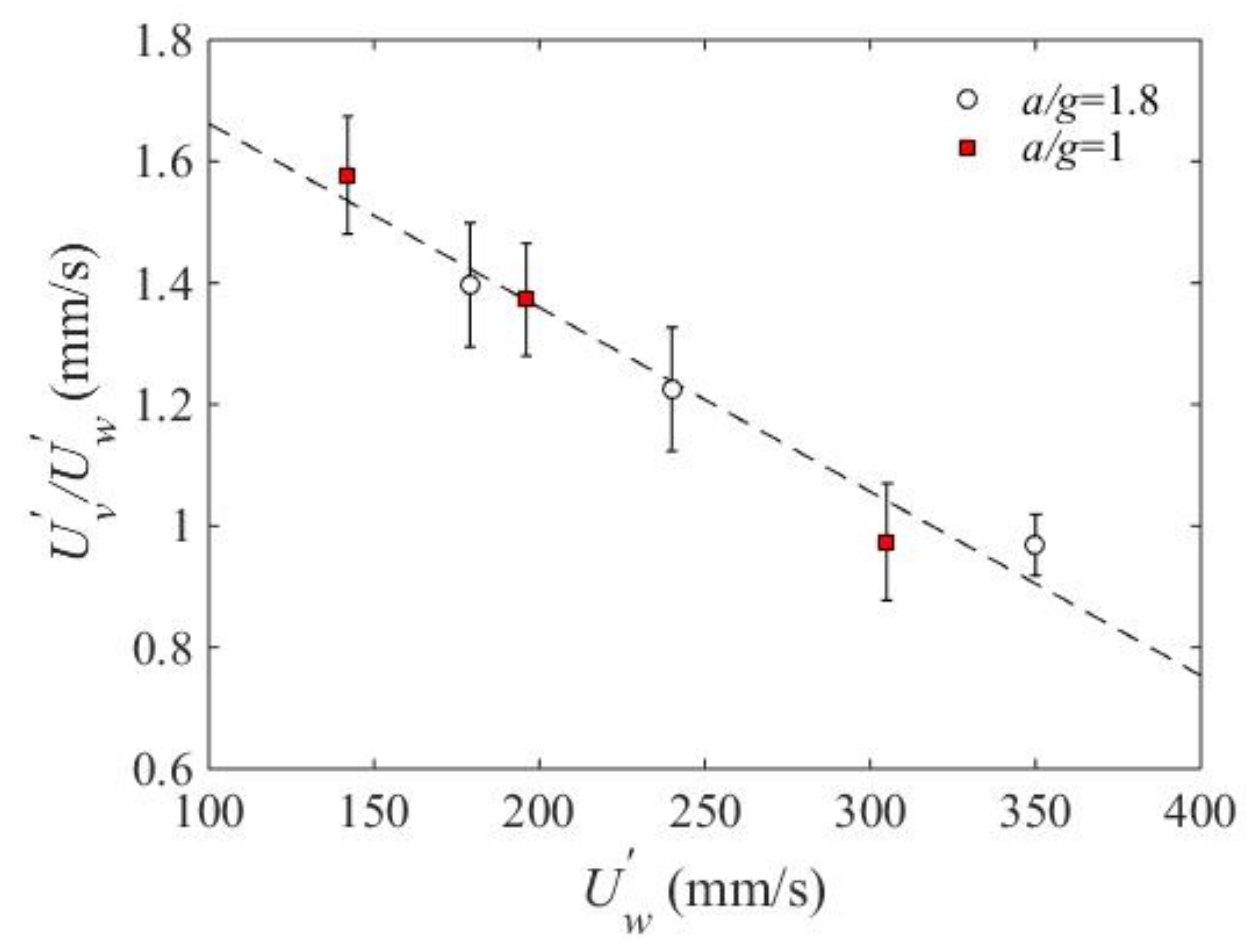

Figure 18: Velocity of vortices as they move away from the bubble tail (fixed reference frame at bubble tail) with respect to bubble velocity at $a / g=1.8$ and $a / g=1$.

The various time-resolved wake characteristics that have been gleaned from Figure 15 and Figure 16, can be used to describe time-averaged heat transfer results. To further compare the effect of drift velocity and gravity on the wake heat transfer, data were averaged with time for 520 bubbles at each test condition to obtain the profiles shown in Figure 19. The near-wake region (Figure 19a) is characterized by the rise of the heat transfer coefficient enhancement behind the bubble tail. The penetration length is seen to increase with $U_{d}$ for both gravity levels, with $a / g=1.8$ exhibiting slightly longer $L_{p}$ than $a / g=1$. Both observations may be attributed to the increase in liquid film momentum with increasing drift velocity and gravity level.

The peak heat transfer enhancement is also seen to vary with drift velocity, generally decreasing with increasing $U_{d}$ at each acceleration. Scammell and Kim [21] theorized that the behavior was due to the reduced residence time of faster moving bubbles over a given position on the heated tube, thereby reducing the ability of vortices to remove heat from the wall. The addition of $1.8 \mathrm{~g}$ acceleration data in the current study, however, seems to disprove this explanation as the enhancement peaks do not strictly decrease with $U_{b}$. It may be noticed that the 
peak behavior over both accelerations appears to coincide with the relative distribution of $L_{p}$ at each drift velocity. At $a / g=1.8, L_{p}$ is seen to vary widely (Figure 16), thereby creating more diffuse and smaller magnitude peaks than $a / g=1$ (Figure 15). Definitive elucidation of these trends requires coupling of local flow field measurements, which were not available in this study.

The far-wake region (Figure 19b) shows a continuation in enhancement decay to the singlephase value far downstream of the bubble. The distance behind the tail at which single-phase heat transfer is re-established (the "calming length", $L_{c} / D$ ) is seen to depend on the liquid Reynolds number and the gravity level. This behavior may be attributed to the redevelopment of the thermal boundary layer in the wake after its disruption by vortices as was shown by Babin et al. [20]. For both $1 \mathrm{~g}$ and $1.8 \mathrm{~g}$ accelerations, $L_{c} / D$ was smallest at $R e_{F} \approx 800$, increased to its largest values at $R e_{l}=1570$, then decreased to moderate lengths at $R e_{l}=3058$. Assuming laminar flow, increasing $R e_{l}$ from approximately 800 to 1570 would have resulted in longer thermal and hydraulic redevelopment lengths. Increasing $R e_{l}$ further to 3080, where the flow was likely transitional, would have caused a decrease in the redevelopment length due to the onset of turbulence. The growth in $L_{c} / D$ with acceleration may be attributed to the increased influence of natural convection at hypergravity $\left(R a_{D, 1.8 g} / R a_{D, 1 g} \approx 2.8,2.1\right.$, and 2.3 for $R e_{l}=800,1570$, and 3090) which effectively increased the Reynolds number near the wall and led to a longer development length. 
a)

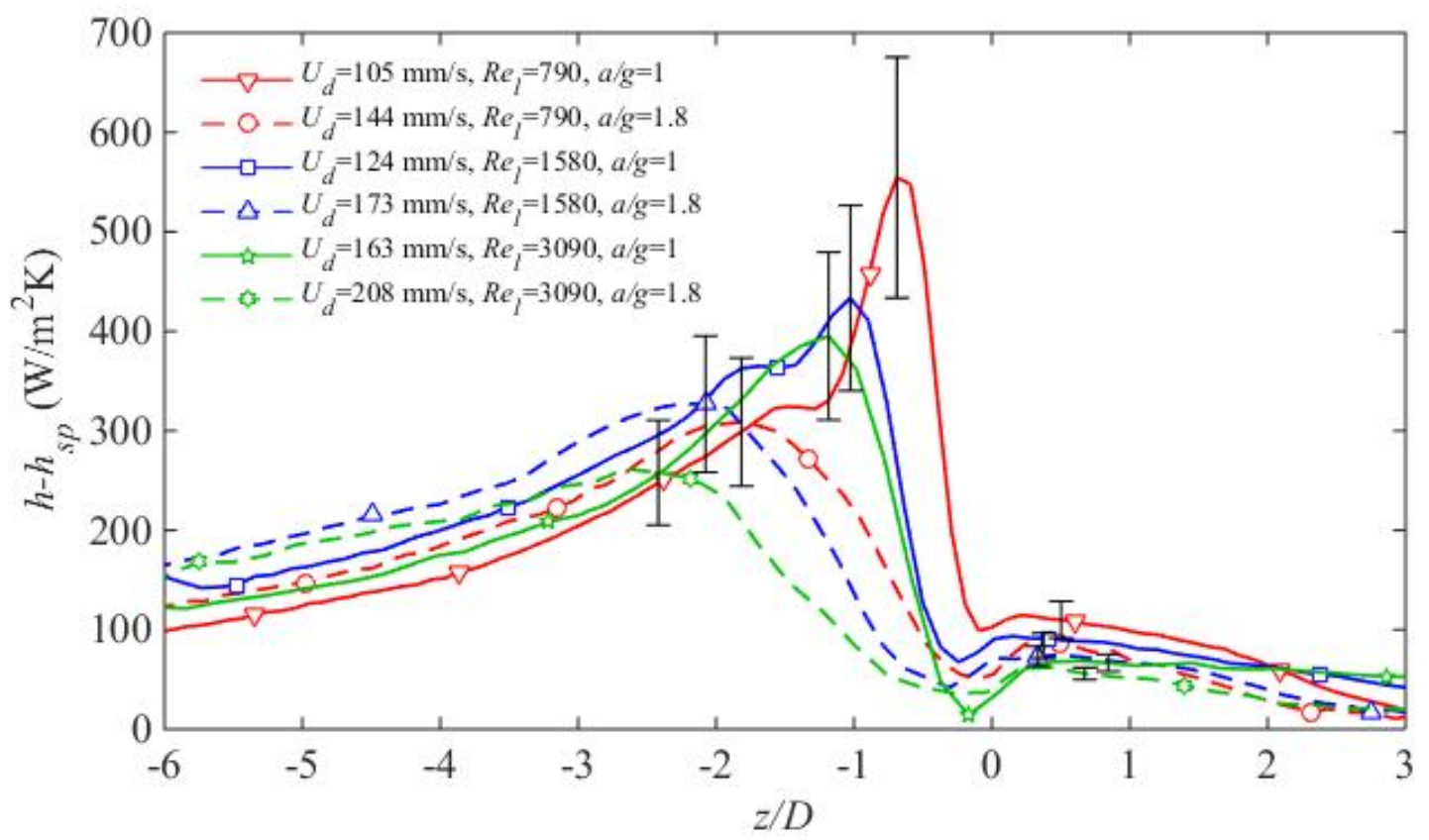

b)

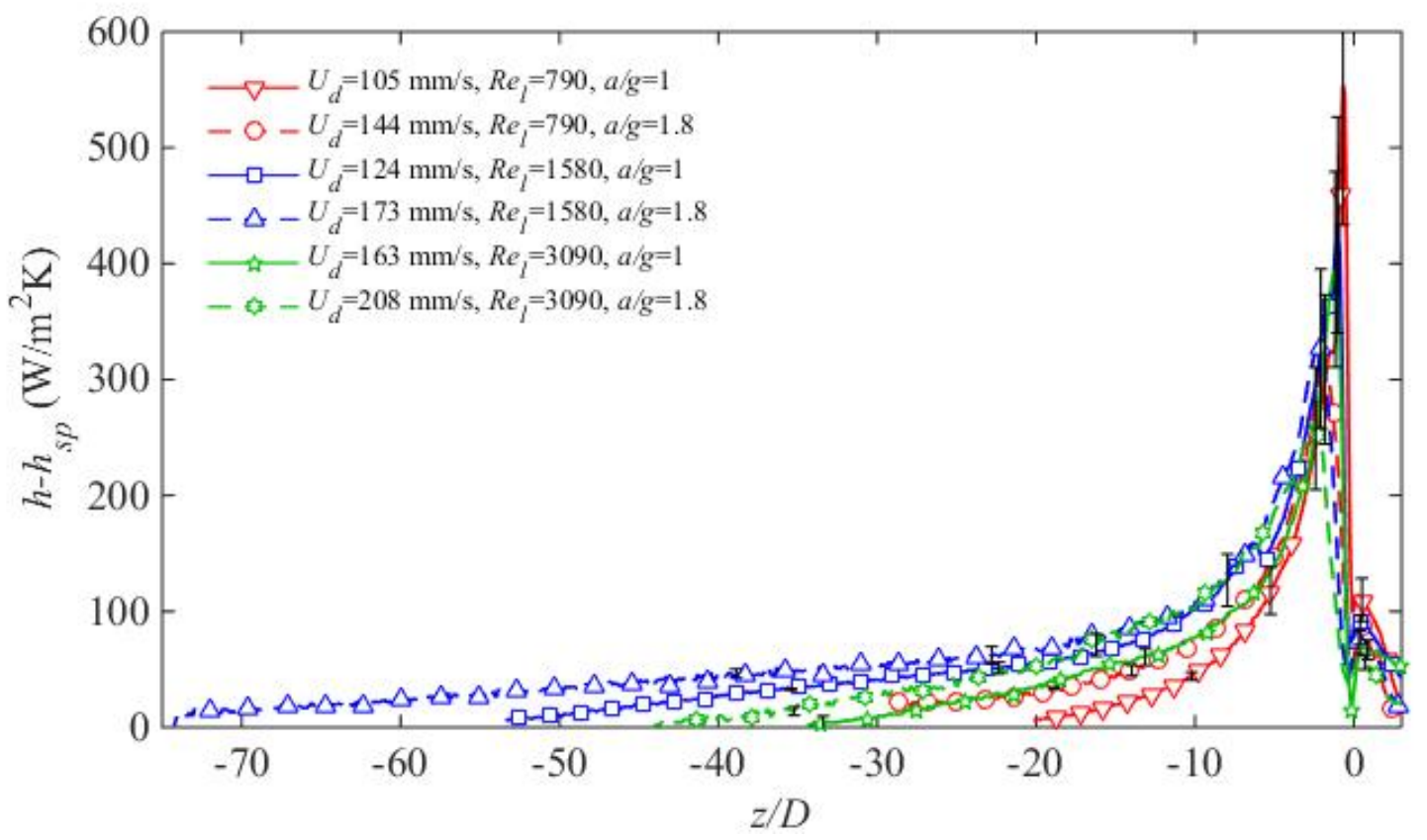

Figure 19: Heat transfer coefficient enhancement as a function of distance behind the bubble tail for a) near-wake region and b) far-wake regions at the conditions: $U_{d}=105,124,163,144,173$, and $208 \mathrm{~mm} / \mathrm{s}$; tube heat flux of $\mathrm{q}^{\prime \prime}=1400,1470,1700,1140,1330$, and $1320 \mathrm{~W} / \mathrm{m}^{2}$, respectively; average single-phase heat transfer coefficient of $h_{S P}=130,148,192,104,112$, and $146 \mathrm{~W} / \mathrm{m}^{2} \mathrm{~K}$, respectively. 


\subsection{Relevance to heat transfer prediction}

Some correlations for flow boiling heat transfer have used factors to account for flow regime evolution (for example: $F$ in Chen [43] and $C_{1}, C_{2}$ in Schrock and Grossman [44]) allowing predictions to be made over a wide range of parameters with relatively straightforward calculations. When compared to data from the literature, however, scatter is often seen in the data with respect to the correlations. Some of the variation is likely due to discrepancies and uncertainties in measurement techniques. Another potential influence is the difference in flow regime for a given set of parameters (liquid mass flux, vapor quality, heat flux, etc.) due to variations in experiments. These include aspects such as tube roughness and the local wall thermal boundary condition. The current work illustrates that under similar flow and heating parameters, elongated bubbles can provide notably different heat transfer coefficient enhancement depending on the observed local hydrodynamics. While cumbersome, it may be necessary for future correlations and models to account for the specifics of the flow regimes expected during heat exchanger operation. Necessary characteristics for slug flow may include the bubble shape, presence of wake turbulence, presence of film nucleation, and bubble growth. Prediction is further complicated when sequential bubbles or bubble trains exist where the bubble frequency and length must be incorporated. Future experimental and numerical results will be most beneficial if the mechanisms of specific flow regimes are identified and the conditions at which the data was obtained are carefully detailed. A database may then be assembled to include both flow regime and heat transfer predictions with the hope of providing more focused and accurate design tools.

\section{CONCLUSIONS}

A study on the heat transfer in the presence of an elongated bubble rising co-currently with vertical, upward flowing liquid was presented. Data collected in the laboratory and on an aircraft flying parabolic trajectories allowed for bubbles characterized by a wide range of Bond numbers to be observed. Visual analysis of the bubbles illustrated the effect of gravity, and subsequently drift velocity on the bubble profile. Bubbles exhibited small drift velocities and shapes characteristic of capillary bubbles at low gravity levels, while terrestrial and hypergravity conditions produced Taylor bubbles moving at higher drift velocities. The measured film 
thicknesses were shown to vary little with acceleration and be in agreement with the correlations of Brown [6] and Llewellin et al. [12].

The flow dynamics induced by the different gravity levels was found to strongly influence the bubble shape and the local heat transfer characteristics. Large heat transfer enhancement was observed behind Taylor bubbles due to the presence of vortices in the wake. Capillary bubbles provided little wake heat transfer enhancement and only slight improvement in the liquid film. The frequency of vortex shedding for Taylor bubbles was shown to increase linearly with the plunging velocity, allowing for the proposal of a critical impinging velocity to predict vortex incipience. Wall jet separation was found to affect the tube wall at differing penetration lengths depending on the acceleration and bubble drift velocity. Time-averaged heat transfer coefficient profiles showed that the variation in penetration length coincides with smaller and broader peaks in wake enhancement for $a / g=1.8$ compared to $a / g=1$.

\section{ACKNOWLEDGEMENTS}

This work was supported by NASA Space Technology Research Fellowship NNX11AN49H and NASA grant NNX09AK39A. The authors would also like to thank Esli Trejo Peimbert (IMFT, Toulouse, France) and Donghyeon Lee (POSTECH, Pohang, South Korea) for their assistance in collecting the variable gravity portion of the data presented. Mirco Magnini was supported by the Swiss National Science Foundation (SNSF) under Contract No. 200020156181.

\section{NOMENCLATURE}

\section{General}

$a$, acceleration $\left(\mathrm{m} / \mathrm{s}^{2}\right)$

$\beta$, coefficient of volumetric expansion $(1 / \mathrm{K})$

$B o$, Bond number

$C$, velocity drift constant

$\mathrm{Ca}$, Capillary number

$D$, tube diameter $(\mathrm{m})$

$f$, frequency $(\mathrm{Hz})$

$g$, gravitational acceleration $\left(\mathrm{m} / \mathrm{s}^{2}\right)$

$G$, liquid mass flux $\left(\mathrm{kg} / \mathrm{m}^{2} \mathrm{~s}\right)$

$\mathrm{Ga}$, Galileo number

$h$, heat transfer coefficient $\left(\mathrm{W} / \mathrm{m}^{2} \mathrm{~K}\right)$

$k$, thermal conductivity $(\mathrm{W} / \mathrm{m}-\mathrm{K})$ 
$L$, length (mm)

$N$, number of samples

$N_{f}$, dimensionless group

$\mathrm{Nu}$, Nusselt number

$P$, pressure $(\mathrm{Pa})$

$q$ ", heat flux $\left(\mathrm{W} / \mathrm{m}^{2}\right)$

$r$, radial position (mm)

$R$, tube radius (mm)

$R e$, Reynolds number

$S t$, Strouhal number

$T$, temperature $\left({ }^{\circ} \mathrm{C}\right)$

$U$, velocity $(\mathrm{mm} / \mathrm{s})$

$z$, axial distance $(\mathrm{mm})$

Greek

$\alpha$, absorptivity

$\delta$, film thickness or uncertainty

$\lambda$, wavelength

$\mu$, dynamic viscosity

$v$, kinematic viscosity

$\rho$, density

$\sigma$, surface tension

$\underline{\text { Subscripts }}$

$a b s$, absolute

$b$, bubble

cam, IR camera

$c$, calming

$c r$, critical

$d$, drift

$f$, film

$K$, Keyence sensor

$l$, liquid

$m$, mean

$p$, plunging, penetration, or polyimide

$r$, random

sat, saturation

$\mathrm{Si}$, Silicon

$S P$, single-phase

$V$, visual camera

$v$, vapor/vortex

$w$, wake or wall

0 , stagnant conditions 


\section{REFERENCES}

[1] Viana, F., Pardo, R., Yánez, R., Trallero, J. L., and Joseph, D. D., 2003, "Universal correlation for the rise velocity of long gas bubbles in round pipes," J. Fluid Mech., 494, pp. 379-398.

[2] Fabre, J., and Line, A., 1992, "Modeling of Two-Phase Slug Flow," Annu. Rev. Fluid Mech., 24(1), pp. 21-46.

[3] Nicklin, D. J., Wilkes, J. O., and Davidson, J. F., 1962, "Two phase flow in vertical tubes," Trans. Inst. Chem. Eng., 40, pp. 61-68.

[4] Collins, R., Moraes, F. F. De, Davidson, J. F., and Harrison, D., 1978, "The motion of a large gas bubble rising through liquid flowing in a tube," J. Fluid Mech., 89, pp. 497-514.

[5] White, E. T., and Beardmore, R. H., 1962, "The velocity of rise of single cylindrical air bubbles through liquids contained in vertical tubes," Chem. Eng. Sci., 17, pp. 351-361.

[6] Brown, R. A. S., 1965, "The mechanics of large gas bubbles in tubes: I. Bubble velocities in stagnant liquids," Can. J. Chem. Eng., 43(5), pp. 217-223.

[7] Rattner, A. S., and Garimella, S., 2015, "Vertical upward intermediate scale Taylor flow: Experiments and kinematic closure," Int. J. Multiph. Flow, 75, pp. 107-123.

[8] Bendiksen, K. H., 1985, "On the motion of long bubbles in vertical tubes," Int. J. Multiph. Flow, 11(6), pp. 797-812.

[9] Campos, J. B. L. M., and Carvalho, J. R. F. G. De, 1988, “An experimental study of the wake of gas slugs rising in liquids," J. Fluid Mech., 196, pp. 27-37.

[10] Nogueira, S., Riethmuler, M. L., Campos, J. B. L. M., and Pinto, a. M. F. R., 2006, "Flow in the nose region and annular film around a Taylor bubble rising through vertical columns of stagnant and flowing Newtonian liquids," Chem. Eng. Sci., 61(2), pp. 845857.

[11] Goldsmith, H. L., and Mason, S. G., 1962, "The movement of single large bubbles in closed vertical tubes," J. Fluid Mech., 14(01), p. 42.

[12] Llewellin, E. W., Del Bello, E., Taddeucci, J., Scarlato, P., and Lane, S. J., 2011, "The thickness of the falling film of liquid around a Taylor bubble," Proc. R. Soc. A Math. Phys. Eng. Sci., 468(2140), pp. 1041-1064.

[13] Liu, Y.-P., Wang, P.-Y., Wang, J., and Du, Z.-H., 2013, "Investigation of Taylor bubble wake structure in liquid nitrogen by PIV technique," Cryogenics (Guildf)., 55-56, pp. 2029.

[14] Pinto, A. M. F. R., Coelho Pinheiro, M. N., and Campos, J. B. L. M., 1998, "Coalescence of two gas slugs rising in a co-current flowing liquid in vertical tubes," Chem. Eng. Sci., 53(16), pp. 2973-2983.

[15] Kawaji, M., DeJesus, J. M., and Tudose, G., 1997, "Investigation of flow structures in vertical slug flow," Nucl. Eng. Des., 175(1-2), pp. 37-48.

[16] Shemer, L., Gulitski, a., and Barnea, D., 2007, "On the turbulent structure in the wake of Taylor bubbles rising in vertical pipes," Phys. Fluids, 19(3), p. 035108.

[17] Hetsroni, G., and Rozenblit, R., 2000, "Thermal patterns on a heated wall in vertical airwater flow," Int. J. Multiph. Flow, 26(2), pp. 147-167.

[18] Babin, V., Barnea, D., and Shemer, L., 2010, "Heat Transfer Characteristics in a Slug Unit," IHTC14-22649, Washington D.C. 
[19] Babin, V., Shemer, L., and Barnea, D., 2012, "Experimental investigation of the local heat transfer in a vertical gas-liquid slug unit," The 7th International Symposium on Measurement Techniques for Multiphase Flows, pp. 43-50.

[20] Babin, V., Shemer, L., and Barnea, D., 2015, "Local instantaneous heat transfer around a rising single Taylor bubble," Int. J. Heat Mass Transf., 89, pp. 884-893.

[21] Scammell, A., and Kim, J., 2015, "Heat transfer and flow characteristics of rising Taylor bubbles," Int. J. Heat Mass Transf., 89, pp. 379-389.

[22] Brauner, N., and Maron, D. M., 1992, "Identification of the range of 'small diameters' conduits, regarding two-phase flow pattern transitions," Int. Commun. Heat Mass Transf., 19(1), pp. 29-39.

[23] Kandlikar, S. G., 2002, "Fundamental issues related to flow boiling in minichannels and microchannels," Exp. Therm. Fluid Sci., 26(2-4), pp. 389-407.

[24] Bretherton, F. P., 1961, "The motion of long bubbles in tubes," J. Fluid Mech., 10(02), pp. 166-188.

[25] Jacobi, A. M., and Thome, J. R., 2002, "Heat Transfer Model for Evaporation of Elongated Bubble Flows in Microchannels," J. Heat Transfer, 124(6), pp. 1131-1136.

[26] Thome, J. R., Dupont, V., and Jacobi, a. M., 2004, "Heat transfer model for evaporation in microchannels. Part I: presentation of the model," Int. J. Heat Mass Transf., 47(14-16), pp. 3375-3385.

[27] Magnini, M., Pulvirenti, B., and Thome, J. R., 2013, "Numerical investigation of hydrodynamics and heat transfer of elongated bubbles during flow boiling in a microchannel," Int. J. Heat Mass Transf., 59, pp. 451-471.

[28] Kim, T. H., Kommer, E., Dessiatoun, S., and Kim, J., 2012, "Measurement of two-phase flow and heat transfer parameters using infrared thermometry," Int. J. Multiph. Flow, 40, pp. 56-67.

[29] Al-Arabi, M., 1982, "Turbulent Heat Transfer in the Entrance Region of a Tube," Heat Transf. Eng., 3(3-4), pp. 76-83.

[30] Narcy, M., Scammell, A., Colin, C., and Kim, J., 2014, "Flow Boiling Under Microgravity Conditions: Comparative study of two experimental data sets," Proceedings of the 15th International Heat Transfer Conference, Kyoto, pp. IHTC15-9072.

[31] Churchill, S. W., and Chu, H. H. S., 1975, "Correlating equations for laminar and turbulent free convection from a vertical plate," Int. J. Heat Mass Transf., 18(11), pp. 1323-1329.

[32] Incropera, F. P., DeWitt, D. P., Bergman, T. L., and Lavine, A. S., 2007, Fundamentals of Heat and Mass Transfer, Wiley.

[33] Shah, R. K., and London, A. L., 1978, "Laminar Flow Forced Convection in Ducts," Advances in Heat Transfer, Academic Press, New York.

[34] Davis, L. P., and Perona, J. J., 1971, "Development of free convection flow of a gas in a heated vertical open tube," Int. J. Heat Mass Transf., 14(7), pp. 889-903.

[35] Taylor, J. R., 1982, An Introduction to Error Analysis, University Science Books.

[36] Colin, C., Fabre, J., and Dukler, A. E., 1991, "Gas-liquid flow at microgravity conditions - I. Dispersed bubble and slug flow,” Int. J. Multiph. Flow, 17(4), pp. 533-544.

[37] Polonsky, S., Barnea, D., and Shemer, L., 1999, "Averaged and time-dependent characteristics of the motion of an elongated bubble in a vertical pipe," Int. J. Multiph. Flow, 25(5), pp. 795-812.

[38] Rasband, W. S., 1997, "ImageJ." 
[39] Dumitrescu, D. T., 1943, "Strömung an einer Luftblase im senkrechten Rohr," ZAMM Zeitschrift für Angew. Math. und Mech., 23(3), pp. 139-149.

[40] Ratulowski, J., and Chang, H.-C., 1989, "Transport of gas bubbles in capillaries," Phys. Fluids A, 1(10), pp. 1642-1655.

[41] Edvinsson, R. K., and Irandoust, S., 1996, "Finite-element analysis of Taylor flow," AIChE J., 42(7), pp. 1815-1823.

[42] Shemer, L., Gulitski, A., and Barnea, D., 2005, "Experiments on the Turbulent Structure and the Void Fraction Distribution in the Taylor Bubble Wake," Multiph. Sci. Technol., 17(1-2), pp. 103-122.

[43] Chen, J. C., 1966, "Correlation for Boiling Heat Transfer to Saturated Fluids in Convective Flow,” Ind. Eng. Chem. Process Des. Dev., 5(3), pp. 322-329.

[44] Schrock, V. E., and Grossman, L. M., 1962, "Forced Convection Boiling in Tubes," Nucl. Sci. Eng., 12(4), pp. 474-481. 